\title{
Functionally Reduced Sensorimotor Connections Form with Normal Specificity Despite Abnormal Muscle Spindle Development: The Role of Spindle-Derived Neurotrophin 3
}

\author{
Neil A. Shneider, ${ }^{1,2,3 \star}$ George Z. Mentis, ${ }^{1 *}$ Joshua Schustak, ${ }^{1}$ and Michael J. O’Donovan ${ }^{1}$ \\ ${ }^{1}$ Section on Developmental Neurobiology, National Institute of Neurological Disorders and Stroke, National Institutes of Health, Bethesda, Maryland 20892, \\ and ${ }^{2}$ Department of Neurology and ${ }^{3}$ Center for Motor Neuron Biology and Disease, Columbia University, New York, New York 10032
}

The mechanisms controlling the formation of synaptic connections between muscle spindle afferents and spinal motor neurons are believed to be regulated by factors originating from muscle spindles. Here, we find that the connections form with appropriate specificity in mice with abnormal spindle development caused by the conditional elimination of the neuregulin1 receptor ErbB2 from muscle precursors. However, despite a modest $(\sim 30 \%)$ decrease in the number of afferent terminals on motor neuron somata, the amplitude of afferent-evoked synaptic potentials recorded in motor neurons was reduced by $\sim 80 \%$, suggesting that many of the connections that form are functionally silent. The selective elimination of neurotrophin 3 (NT3) from muscle spindles had no effect on the amplitude of afferent-evoked ventral root potentials until the second postnatal week, revealing a late role for spindle-derived NT3 in the functional maintenance of the connections. These findings indicate that spindle-derived factors regulate the strength of the connections but not their initial formation or their specificity.

\section{Introduction}

The mechanisms regulating the formation of synaptic connections between muscle spindle afferents and spinal motor neurons have been the subject of considerable study (Chen et al., 2003; Ladle et al., 2007). In adult mammals, spindle afferents make monosynaptic connections with homonymous and synergistic motor neurons but not with functionally antagonistic motor neurons. There is now a consensus that signals derived from the periphery are critical for the specification and functional development of these connections, and recent work has focused on the role of the muscle spindle and spindle-derived factors in this process (Frank and Wenner 1993; Wenner and Frank, 1995; Chen et al., 2003). It has been shown that the gross anatomical projection pattern of muscle afferents is normal in Egr3 ${ }^{-1-} \mathrm{mu}-$ tants in which the spindles degenerate after birth (Tourtellotte and Milbrandt, 1998; Tourtellotte et al., 2001; Chen et al., 2002) and in the ErbB2 ${ }^{-1-}$ mutant in which spindle induction is severely compromised (Leu et al., 2003). However, no study has examined physiologically or anatomically the projections of muscle-identified afferents onto individual identified motor

\footnotetext{
Received Dec. 4, 2008; revised March 2, 2009; accepted March 4, 2009.

This work was supported by the intramural program of the National Institute of Neurological Disorders and Stroke. N.A.S. was also supported by Project ALS and by a New York State Department of Health Spinal Cord Injury research grant (Contract C018610). We thank Monica Mendelsohn and Barbara Han for expert technical assistance in the generation of the Egr3-IRES-Cre mouse line and Hua Tian for her invaluable technical assistance. Special thanks to Tom Jessell and Chris Henderson for their support and critical comments on this manuscript.

${ }^{*}$ N.A.S. and G.Z.M. contributed equally to this work.

Correspondence should be addressed to MichaelJ. O'Donovan, Section on Developmental Neurobiology, National Institute of Neurological Disorders and Stroke, National Institutes of Health, Bethesda, MD 20892. E-mail: odonovm@ninds.nih.gov.

DOI:10.1523/JNEUROSCI.5790-08.2009

Copyright $\odot 2009$ Society for Neuroscience $\quad$ 0270-6474/09/294719-17\$15.00/0
}

neurons in animals with abnormal muscle spindle development. As a result, the role of the muscle spindle in determining the specificity of these connections is unknown.

One spindle-derived signaling molecule that has been implicated in regulating the strength of sensorimotor connections is neurotrophin-3 (NT3) (Chen et al., 2002). NT3 is first expressed in both extrafusal and intrafusal muscle fibers but becomes restricted to intrafusal muscles in the postnatal period (Copray and Brouwer, 1994; Wright et al., 1997). Analysis of Egr $3^{-1-}$ mice has revealed that monosynaptic ventral root potentials evoked by muscle nerve stimulation fail as early as postnatal day 2 (P2), but they can be subsequently rescued bilaterally by the chronic injection of NT3 protein into a single postnatal hindlimb (Chen et al., 2002). However, because NT3 is known to potentiate proprioceptive sensory inputs to motor neurons both acutely (Arvanov et al., 2000; Arvanian and Mendell, 2001) and chronically (Arvanian et al., 2003), it remains possible that exogenous NT3 compensates pharmacologically for some other aspect of spindle development that is abnormal in the Egr3 knock-out mutant.

In the present work, we have examined the strength and specificity of muscle afferent projections to identified motor neurons in mice in which muscle spindle induction is inhibited by the conditional elimination of the neuregulin 1 (Nrg1) receptor ErbB2 from muscle spindle precursors. We show that the synaptic connections between muscle afferents and motor neurons form with normal specificity but are functionally weaker than their wild-type (WT) counterparts during the first postnatal week. We also find that targeted elimination of NT3 from muscle spindles has no effect on the amplitude of dorsal root-evoked ventral root potentials during the first postnatal week. Collectively, these results suggest that the specificity of sensorimotor 
connections is regulated independently of the muscle spindle and indicate that spindle-derived factors other than NT3 regulate the strength of the connections in the first postnatal week.

\section{Materials and Methods}

Generation of Egr $3^{C R E}$ mice. Mouse Egr3 genomic clones from a 129sv/J genomic library were kindly provided by Warren Tourtellotte, Northwestern University, Feinberg School of Medicine, Chicago, IL (Tourtellotte and Milbrandt, 1998). The targeting vector for the Egr $3{ }^{\text {Cre }}$ allele was constructed from a $7.2 \mathrm{~kb}$ BamHI/HindIII fragment containing the Egr3 zinc finger domain coding sequence. A Bam HI site immediately $3^{\prime}$ to the Egr3 stop codon was mutated to a PacI site, into which was cloned a $4 \mathrm{~kb}$ internal ribosomal entry site (IRES)-Cre-simian virus $40 \mathrm{pA} / \mathrm{TK}-$ neo-pA PacI cassette (provided by Silvia Arber, University of Basel, Basel, Switzerland). The linearized targeting construct was electroporated into W9.5 embryonic stem (ES) cells, selected with G418, and screened for homologous recombinants by Southern analysis (HindIII digest) using as probe a $500 \mathrm{bp} \mathrm{BamHI}$ fragment upstream of the $5^{\prime}$ end of the targeting construct. The frequency of recombination with this construct was $\sim 15 \%$. Recombinant clones were injected into C57BL/6J blastocysts to generate chimeric founders. The Egr3 ${ }^{\mathrm{CRE}}$ allele was maintained on a predominantly C57BL/6J strain background.

Other mouse lines used in this study were obtained from the following sources: NT3 ${ }^{\text {FLOX }}$ (Bates et al., 1999) from The Jackson Laboratory (strain number 003541; B6.129S4-Ntf3 ${ }^{\text {tm2Jae }}$ ); NT3 ${ }^{\text {LacZ }}$ mice (Fariñas et al., 1994) from the Mutant Mouse Regional Resource Centers (www. mmrrc.org) (stock number 000191-UCD; Ntf3 ${ }^{\text {tmlLfr }} / \mathrm{Mmcd}$ ); $E r b B 2^{N U L L}$ (Lee et al., 1995) and ErbB2 ${ }^{\text {FLOX }}$ (Crone et al., 2003) from Kuo-Fen Lee (Salk Institute, La Jolla, CA); $E g r 3^{N U L L}$ null from Warren Tourtellotte (Tourtellotte et al., 2001); and myf5 ${ }^{C R E}$ (Tallquist et al., 2000) from Philippe Soriano (Fred Hutchinson Cancer Research Center, Seattle, WA). Wild-type mice were obtained from The Jackson Laboratory, Charles River, or Taconic Farms.

In situ hybridization. In situ hybridization analysis was performed with digoxigenin-labeled cRNA probes (Schaeren-Wiemers and GerfinMoser, 1993) specific for Egr3 (Hippenmeyer et al., 2002), Er81 (Arber et al., 2000), and NT3. The NT3 probe, corresponding to the entire NT3 coding sequence, was amplified by PCR from genomic DNA isolated from myogenin-NT3 transgenic animals (Wright et al., 1997).

Muscle spindle counts. Muscle spindle counts were performed by in situ hybridization analysis, as above. Tibialis anterior (TA) muscles were dissected from each mouse hindlimb at birth (P0) or P3 and cryosectioned at a thickness of $18 \mu \mathrm{m}$. All sections from an individual TA muscle were analyzed using riboprobes for Egr3, and all sections from the contralateral TA muscle were analyzed with a probe for ER81. The total number of Egr3-positive $\left(\mathrm{Egr}^{+}{ }^{+}\right)$or ER81 ${ }^{+}$muscle spindles was counted under low magnification, with special attention given to the relative position and orientation of individual spindles on contiguous sections to avoid counting a single muscle spindle more than once. No significant difference in spindle numbers was observed between the ErbB2 $2^{\text {NULL/FLOX }}$ and ErbB2 ${ }^{\text {FLOX/FLOX }}$ control animals, and data from these animals were combined.

Immunohistochemistry. Spinal cords were dissected from animals that were transcardially perfused with PBS, followed by $4 \%$ paraformaldehyde (PFA), fixed overnight in 4\% PFA, and subsequently transferred to PBS. Some spinal cords were processed for immunostaining after electrophysiological recordings or were used for orthograde and anterograde tracing in vitro, in which case they were only submersion fixed overnight in $4 \%$ PFA. Spinal cords were embedded in warm $5 \%$ agar, and serial transverse sections $(70 \mu \mathrm{m})$ were cut with a vibratome. The sections were collected in wells and processed for the vesicular glutamate transporter 1 (VGluT1) immunostaining. For neonatal (P1-P7) spinal cords, the rabbit polyclonal VGluT1 antibody used was obtained from Synaptic Systems; for P21 spinal cord, the guinea pig polyclonal VGluT1 antibody used was generated and characterized by Julia Kaltschmidt (Jessell laboratory, Columbia University, New York, NY). Sections were also stained for choline acetyltransferase (ChAT) with a goat polyclonal (AB144) from Millipore.
All spinal cord sections were processed as free-floating sections to allow antibody access to both sides of the section. The sections were blocked with $10 \%$ normal donkey serum in $0.01 \mathrm{~m}$ PBS with $0.1 \%$ Triton $\mathrm{X}-100$ (PBS-T), $\mathrm{pH}$ 7.4, and incubated overnight at room temperature in VGluT1 antiserum diluted in PBS-T. The following day, secondary immunostaining was performed for $3 \mathrm{~h}$ with rabbit antiserum (secondary antibody) coupled to either FITC (The Jackson Laboratory), Alexa 488, or Alexa 647 (Invitrogen) depending on the experiment. After secondary antibody incubations, the sections were washed in PBS. All fluorescent preparations were mounted in an anti-fading solution glycerol/PBS (3:7) or Vectashield (Vector Laboratories).

For muscle spindle analysis, postnatal hindlimbs were fixed overnight in $4 \%$ PFA, washed in PBS, cryopreserved in 30\% sucrose $/ 0.1 \mathrm{M}$ phosphate buffer, and then frozen in O.C.T. (Pro SciTech). Cryostat sections were cut at a thickness of $14-20 \mu \mathrm{m}$ and placed on glass slides for immunostaining using the same conditions as above. Sections were stained with a rabbit (PV28) or goat (PVG 214) polyclonal against parvalbumin (PV) (Swant) and the guinea pig anti-VGluT1 polyclonal (J. Kaltschmidt, as above).

To preserve the morphology of muscle spindles in an intact muscle, whole-mount staining of the gluteus maximus muscle was performed. Hindlimbs were immersion fixed briefly for $\sim 10 \mathrm{~min}$ in $4 \%$ PFA before the gluteus was dissected free. The isolated muscle was then processed by the method used for free-floating vibratome sections of spinal cord, as above. After extensive washing ( $1 \mathrm{~h}$ in PBS-T five times at room temperature and then overnight in $\mathrm{PBS}$ at $4^{\circ} \mathrm{C}$ ), the intact gluteus maximus muscle was mounted in glycerol/PBS (3:7), and spindles were imaged in this muscle using a Zeiss Lumar fluorescent stereo microscope.

Retrograde labeling of muscle-identified motor neurons. Motor neurons supplying the TA and the lateral gastrocnemius (LG) muscles were retrogradely labeled in vivo by the intramuscular injection of cholera toxin B subunit (CTb) conjugated to Alexa 488 and Alexa 647, respectively. For this purpose, mice were anesthetized by halothane inhalation. Two small incisions in the front and lateral sides of the hindlimb were performed to expose the TA and LG muscles, respectively. Each muscle was injected at multiple sites with a total of 2-3 $\mu \mathrm{l}$ of $1 \%$ CTb-Alexa 488 (for TA) or CTb-Alexa 647 (for LG) (dissolved in PBS), using a pulled glass microelectrode with a broken tip. A measured volume of CTb was loaded into the glass electrode by suction and was delivered to the muscle using slight positive pressure. To minimize the possibility of dye leakage from the injection site to other muscle, we closed the incision by application of a drop of Vetbond over the first muscle injected before proceeding to the next. Once the skin incisions were closed or glued, the pup was allowed to recover from anesthesia and returned to its cage. All surgical procedures were performed on postnatal mice in accordance with the National Institutes of Health Guidelines on the Care and Use of Animals and approved by the National Institute of Neurological Disorders and Stroke animal care and use committee.

The labeled motor neurons were visualized and targeted with patch electrodes during the physiological experiment that was performed 2-3 d later. Despite our precautions, we found that a small proportion of motor neurons were labeled with both $\mathrm{CTb}-488$ and $\mathrm{CTb}-647$, presumably because of spillover of the injected fluorochrome from one muscle to the other. We did not target these motor neurons for recording.

Anterograde labeling of muscle afferents. To examine the anatomical pattern of muscle afferent projections onto motor neurons, CTb-488 was injected into tibialis anterior muscle and CTb-647 was injected into the lateral gastrocnemius muscle as described above for the labeling of motor neurons. To minimize the possibility of dye leakage and subsequent double labeling, in four animals (two WT, two ErbB2 $\Delta$ ), we injected the muscles at P7, and in two additional animals (one WT, one ErbB2 $\Delta$ ), we staggered the injections into the two muscles. The first muscle was injected at P7, and $1.5 \mathrm{~d}$ later, the antagonistic muscle was injected. In half of the animals, the LG was injected first followed by the $\mathrm{TA}$, and in the other half of the animals, the sequence was reversed. To enhance the probability of visualizing primary afferent boutons, we increased the concentration of CTb from $1 \%$ (used to label motor neurons) to $5 \%$. Pups were killed at P14, which is the earliest age the ErbB2 $\Delta$ mutants manifest the proprioceptive phenotype. The spinal cord was 
dissected free under in vitro conditions at $16^{\circ} \mathrm{C}$ and continuously superfused with artificial CSF (aCSF). The cord was hemisected to improve oxygenation, and the ventral roots L4 and L5 were positioned in suction electrodes and backfilled with Texas Red Dextran [10,000 molecular weight (MW)] for $24 \mathrm{~h}\left(\right.$ at $\left.16^{\circ} \mathrm{C}\right)$ to label motor neurons. This was necessary because motor neuron labeling with $\mathrm{CtB}$ is punctate and the tracer does not continuously fill dendrites. This is in contrast to labeling with dextrans in vitro, which results in dendrites continuously filled with the tracer. After leaving the preparation for $24 \mathrm{~h}$ to fill, the L3-L6 region was excised and fixed in $4 \%$ paraformaldehyde (by immersion fixation) for $24 \mathrm{~h}$. The spinal cord was embedded in $5 \%$ warm agar and subsequently sectioned $(75 \mu \mathrm{m})$ using a vibratome. The sections were collected in wells and processed further for immunohistochemistry against VGluT1 to label the synapses on primary afferents. Finally, sections were mounted on glass slides and coverslipped using an antifading agent (PBS/glycerol, 7:3) until examination with confocal microscopy.

The spinal cord-peripheral nerves preparation. Thirty-seven 2- to 5-dold (P2-P5) mice (18 wild type, 19 ErbB2 $\Delta$ ) (genotype: ErbB2 NULL/FLOX; myf5CRE ${ }^{+/-}$) were used for this part of the study. The mean age was not significantly different between wild type and ErbB2 $\Delta$ mutants (wild type, $3.7 \pm 0.2 \mathrm{~d}$; ErbB2 $\Delta, 4.2 \pm 0.1 \mathrm{~d} ; p=0.32$, Mann-Whitney rank sum test). The animals were decapitated, and the spinal cords were dissected and removed under cold $\left(\sim 12^{\circ} \mathrm{C}\right)$ aCSF containing the following (in mM): $128.35 \mathrm{NaCl}, 4 \mathrm{KCl}, 0.58 \mathrm{NaH}_{2} \mathrm{PO}_{4} \cdot \mathrm{H}_{2} \mathrm{O}, 21 \mathrm{NaHCO}_{3}, 30$ D-glucose, $1.5 \mathrm{CaCl}_{2} \cdot \mathrm{H}_{2} \mathrm{O}$, and $1 \mathrm{MgSO}_{4} \cdot 7 \mathrm{H}_{2} \mathrm{O}$. In some preparations ( 12 wild type and $13 \operatorname{ErbB} 2 \Delta$ ), the sciatic nerve was dissected in continuity with its parent ventral and dorsal roots (L4-L5), after which all the ventral roots were cut. The spinal cord was then transferred to a customized recording chamber located under the objective of a confocal microscope (Axioscope 2 FS MOT, LSM510; Zeiss) equipped with three single photon $(488,543$, and $650 \mathrm{~nm}$ ) and a two-photon laser (Chameleon XR; Coherent) and perfused continuously with oxygenated $\left(95 \% \mathrm{O}_{2} /\right.$ $\left.5 \% \mathrm{CO}_{2}\right)$ aCSF $(\sim 13 \mathrm{ml} / \mathrm{min})$. Ventral roots, dorsal root ganglia (DRG), and cut nerves were placed into suction electrodes for stimulation or recording (see Fig. 4). The peripheral nerves included the deep peroneal nerve supplying the tibialis anterior and extensor digitorum longus muscles (hereafter referred to as the TA nerve), the superficial peroneal nerve supplying the peroneus longus and brevis (SP nerve), the first muscular branch of the tibial nerve supplying the lateral head of gastrocnemius, soleus, and plantaris muscle (LG nerve), and the third muscular branch of the tibial nerve supplying the flexor hallucis longus, tibialis posterior and flexor digitorum longus, and the lateral and medial plantar nerves (tibial nerve). The second muscular branch of the tibial nerve supplying the medial gastrocnemius muscle was excluded because it was easy to damage during the dissection.

Whole-cell recordings from visually identified, retrogradely labeled motor neurons. Whole-cell recordings were obtained with patch electrodes (1.5-2.5 $\mu \mathrm{m}$ tip diameter; $4.4 \pm 0.1 \mathrm{M} \Omega$ average resistance) advanced through the lateral aspect of the spinal cord. The electrode solution contained the following (in $\mathrm{mM}$ ): $10 \mathrm{NaCl}, 130 \mathrm{~K}$-gluconate, $10 \mathrm{HEPES}, 11$ EGTA, $1 \mathrm{MgCl}_{2}, 0.1 \mathrm{CaCl}_{2}$, and $1 \mathrm{Na}_{2} \mathrm{ATP}$, $\mathrm{pH}$ adjusted to $7.2-7.3$ with $\mathrm{KOH}$. Alexa 543 hydrazide $(70 \mu \mathrm{M})$ was added for intracellular labeling of recorded motor neurons (the final osmolarity of the solution was $\sim 305$ $309 \mathrm{mOsm}$ ).

Motor neurons were visually targeted (see Fig. $4 B-D$ ) after removal of the dura and pia mater from the lateral aspect of the cord over the L3-L6 spinal segments. The average distance from the lateral surface of the cord was $165.9 \pm 9.1 \mu \mathrm{m}$ to the recorded TA motor neurons $(n=9)$ and $145.7 \pm 11.5 \mu \mathrm{m}$ for the recorded LG motor neurons $(n=15)$. This reflects the fact the TA motor neuron pool is dorsal to the LG pool. The patch electrode was controlled by a motorized four-axis micromanipulator (Siskiyou) and was advanced toward the motor neuron nucleus rapidly initially and then more slowly (final rate, $\sim 0.2 \mu \mathrm{m} / \mathrm{s}$ ) once motor neurons were encountered as indicated by the presence of an antidromic field potential evoked by ventral root stimulation. Positive pressure was applied to the electrode during the initial rapid advance and "locked" until an increase in tip resistance was registered. Single or two-photon excitation (when depth of pool was $>70 \mu \mathrm{m}$ ) was used to reveal the location of the electrode tip and the motor neuron to which it was at- tached. At this stage, a gigaohm seal was made (cell attached) (see Fig. $4 B$ ), and shortly thereafter slight negative pressure was applied to rupture the cell membrane. The cell was usually labeled by the intracellular dye within 5-10 min, and electrophysiological recordings were performed only if the cell was colabeled with one of the retrogradely transported $\mathrm{CTb}$ dyes (see Fig. 4C,D). Motor neurons labeled with both retrogradely injected dyes were not targeted for electrophysiological recording.

The identity of the recorded cell as a motor neuron was confirmed by evoking an antidromic action potential by stimulation of the cut ventral root. Motor neurons were accepted for additional analysis only if the following three criteria were met: (1) resting membrane potential of -50 $\mathrm{mV}$ or more negative, (2) an overshooting antidromically evoked action potential (see Fig. 4 E), and (3) at least $30 \mathrm{~min}$ of recording. The mean resting membrane potential for all of the motor neurons recorded in this study $(n=22)$ was $-60.8 \pm 1.0 \mathrm{mV}$ (range, -51 to $-71 \mathrm{mV}$ ) after correction for liquid junction potentials $(-7 \mathrm{mV})$. All measurements of synaptic potentials were made at $-60 \mathrm{mV}$ using continuous current injection as necessary. The average recording time per motor neuron $(n=22)$ was $\sim 2 \mathrm{~h}(110.5 \pm 11.8 \mathrm{~min})$.

Synaptic potentials (filtered from DC to $3 \mathrm{kHz}$ ) were recorded from individual motor neurons (Multiclamp 700A; Molecular Devices) in response to orthodromic stimulation (A365, current stimulus isolator; WPI) of muscle nerves or a dorsal root ganglion (L4 or L5). The stimulus threshold (T) was defined as the current at which the minimal evoked response was recorded in three of five trials. The nerves or ganglion were stimulated at 1.2, 1.5, 2, and $5 \times \mathrm{T}$. Recordings were fed to an analog-todigital interface (Digidata 1320A; Molecular Devices) and acquired with Clampex (version 9; Molecular Devices) at a sampling rate of $10 \mathrm{kHz}$. Data were analyzed offline using Clampfit (Molecular Devices). Measurements were made on averaged traces (3-10 trials).

Measurement of motor neuron input resistance. The passive membrane properties of the intracellularly recorded motor neurons were assessed by injection of depolarizing and hyperpolarizing current pulses $(100 \mathrm{~ms}$ duration) at $-60 \mathrm{mV}$ membrane potential. The input resistance was calculated from the slope of the current/voltage plot within the linear range (for the linear range, the membrane potential ranged $\pm 10 \mathrm{mV}$ from the holding potential).

Extracellular recording of afferent-evoked dorsal and ventral root and intraspinal field potentials. Extracellular recordings [Cyberamp (Molecular Devices); amplified $1000 \times$ and filtered from DC to 1 or $3 \mathrm{kHz}$ ] were obtained from the dorsal root ganglia and ventral roots L4 and L5 in response to stimuli ( $0.2 \mathrm{~ms}$ duration) from either of the peripheral nerves, the sciatic nerve en passant or the dorsal root ganglia (L4 or L5) en passant delivered by a stimulus isolator (A365; WPI). The synaptic responses were examined at three different stimulus frequencies $(0.1,1$, and $10 \mathrm{~Hz}$ ) at each stimulation site as part of the "jitter" test of the onset of the EPSP. Recordings were fed to an analog-to-digital interface (Digidata 1320A; Molecular Devices) and acquired with Clampex (version 9; Molecular Devices). Extracellular recordings were sampled at $10 \mathrm{kHz}$, and the recordings were analyzed offline using Clampfit (Molecular Devices). Offline analysis was performed by averaging 3-10 traces (spurious spontaneous events were excluded from the averages). The latency of the afferent-evoked ventral root potentials was defined as the time from the onset of the stimulus artifact to the first measurable deflection of the potential from the baseline.

Intraspinal terminal field potentials (Lev-Tov and Pinco, 1992; Li and Burke, 2001, 2002) were recorded extracellularly using glass microelectrodes, similar to those used for patch recordings, with a tip diameter of $\sim 5 \mu \mathrm{m}$ and a resistance of $0.7 \pm 0.07 \mathrm{M} \Omega$. The internal solution contained $2 \mathrm{M} \mathrm{NaCl}$ plus $0.1 \%$ Fast Green to visualize the electrode. The electrode tip was positioned at the boundary of L4 and L5 (see Fig. 4A) and advanced to the site of the maximal antidromic field potential after stimulation of the L5 ventral root. When positioned in this manner, the electrode sampled the terminal field potentials generated by stimulation of either TA or the LG muscle afferents. Detection of measurable and consistent field potentials required averaging of 250 trials in response to DRG or muscle nerve stimulation at $10 \mathrm{~Hz}$ and $5-10 \times \mathrm{T}$ stimulus intensity. To differentiate between the terminal field potential, representing 
invasion of action potentials into afferent fibers, and the afferent-evoked synaptic field potential of motor neurons, we repeated the averages in low calcium $(0.1 \mathrm{~mm})$ aCSF to block synaptic transmission. Data were acquired after 15-20 min in $0.1 \mathrm{~mm}$ calcium-aCSF (see Fig. 5A).

Calculation of conduction velocity and synaptic delay. The conduction velocity of the primary afferent fibers was calculated from the latencies to the onset of the en passant recording from the DRG after stimulation of the peripheral nerve or the onset of intracellular response after DRG stimulation. At the end of each experiment, the distance from the location of the peripheral nerve stimulating electrode to the DRG was measured with a fine thread. The distance from the DRG to the motor neuron was approximated as the distance from the DRG to the exit point of the ventral root. Only P4 preparations were included in this part of the study (four wild-type and four Erb2 $\Delta$ preparations).

Intracellular filling and immunohistochemistry. All intracellularly recorded neurons were labeled with the fluorescent dye Alexa 568 hydrazide (Invitrogen), which was included in the electrode solution. After the recording session, the spinal cord was fixed in $4 \%$ paraformaldehyde overnight and subsequently transferred to PBS. Spinal cords were embedded in warm $5 \%$ agar, and serial transverse sections ( $70 \mu \mathrm{m})$ were cut with a vibratome. The sections were collected in wells and processed for both the intracellular dye and the retrograde label. We also processed some of the tissue for VGluT1 (raised in rabbit; Synaptic Systems) to label the presynaptic boutons of muscle spindle afferents, as above. Triplelabeled sections were imaged in an LSM510 Zeiss confocal microscope at high magnification $(63 \times)$.

Dorsal and ventral root tracing in the neonatal spinal cord. After the electrophysiological extracellular recordings, the spinal cord was transferred to a dissection chamber, and the L5 ventral and dorsal roots used for physiology were placed in separate suction electrodes and backfilled with the fluorescent dextrans $(30-40 \mathrm{~mm})$ Cascade Blue Dextran $(10,000$ MW; Invitrogen) to label motor neurons and Texas Red Dextran (10,000 MW; Invitrogen) to label sensory axons. Spinal cords from animals younger than P7 were used for labeling without additional dissection, but older cords were hemisected to improve oxygenation. During the labeling procedure, which was performed overnight, the cord was perfused with cold $\left(\sim 16^{\circ} \mathrm{C}\right)$, oxygenated $\left(95 \% \mathrm{O}_{2} / 5 \% \mathrm{CO}_{2}\right)$ aCSF (in mm: 128.35 $\mathrm{NaCl}, 4 \mathrm{KCl}, 0.58 \mathrm{NaH}_{2} \mathrm{PO}_{4} \cdot \mathrm{H}_{2} \mathrm{O}, 21 \mathrm{NaHCO}_{3}, 30$ D-glucose, $0.1 \mathrm{~mm}$ $\mathrm{CaCl}_{2} \cdot \mathrm{H}_{2} \mathrm{O}$, and $2 \mathrm{~mm} \mathrm{MgSO}{ }_{4} \cdot 7 \mathrm{H}_{2} \mathrm{O}$ ). The following day, the cord was immersion fixed in $4 \%$ paraformaldehyde $(4 \mathrm{~h})$ and washed in $0.01 \mathrm{M}$ PBS. Either transverse sections $(70 \mu \mathrm{m})$ were cut from the L5 spinal segment using a vibratome and processed for free-floating immunohistochemistry or alternatively transverse sections $(20 \mu \mathrm{m})$ were cut in a cryostat and processed on slides for subsequent immunocytochemical analysis.

VGluT1 contact counts. Quantitative analysis of VGluT1immunoreactive (IR) $\left(\mathrm{VGluT1}^{+}\right)$synaptic densities on motor neurons at postnatal day 21 were performed on z-series of $1.0 \mu \mathrm{m}$ optically scanned confocal images obtained at high magnification $(63 \times, 1.4 \mathrm{nu}-$ merical aperture, $1.0 \times$ optical zoom,) through the whole cell body and proximal dendrites of large diameter ChAT-IR motor neurons. VGluT1-IR contacts were counted over each motor neuron surface using the Zeiss LSM Image Browser. Contact densities were estimated by measuring the diameter of each motor neuron cell body in all three planes ( $a$, $b, c)$ and calculating the surface area (SA) of each cell, modeled as an ellipsoid, according to Achim Flammenkamp (1990) [www.numericana. com. Surface Area of an Ellipsoid (http://home.att.net/ numericana/ answer/ellipsoid.htm)]. Estimates of soma surface areas were validated by comparing this calculated value with estimates of surface area from three-dimensional renderings of motor neuron somata from the same confocal images using the imaging software Volocity (Improvision). We found no significant differences between somata surfaces estimated with either method (data not shown).

\section{Results}

\section{Inhibition of muscle spindle induction by the conditional elimination of ErbB2}

We first sought to investigate the dependence of the central connectivity of muscle spindle afferents on the muscle spindle. Be- cause neuronal induction of muscle spindle development depends on Nrg1 signaling (Hippenmeyer et al., 2002) through the activation of ErbB2 receptors on primary myocytes (Andrechek et al., 2002; Leu et al., 2003), we generated mice in which ErbB2 expression was eliminated from intrafusal muscle precursors using two targeted alleles of the ErbB2 gene [a null (ErbB2 ${ }^{\mathrm{NULL}}$ ) (Lee et al., 1995) and a conditional (ErbB2 ${ }^{\mathrm{FLOX}}$ ) (Crone et al., 2003) allele] and the deleter line (myf5 ${ }^{\text {CRE}}$ ) (Tallquist et al., 2000) in which the Cre recombinase is expressed in myoblast precursors of intrafusal muscle before the arrival of sensory axons at embryonic day 15 (E15). At approximately postnatal day 15, the $E r b B 2^{\text {NULL/FLOX } / m y f 5} 5^{C R E}$ mutant (referred to as ErbB2 $\Delta$ in the subsequent text and figures) develops a motor phenotype characterized by a flexed posture when inverted and a wide-based, ataxic gait, similar to previously generated mutants that lack muscle ErbB2 (Andrechek et al., 2002; Leu et al., 2003). The

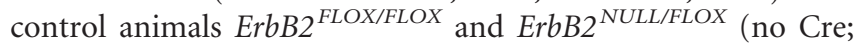
referred to collectively as ErbB2 control) demonstrated no motor abnormality.

When ErbB2 expression is selectively eliminated from muscle, mutant spindles form that are characterized by a single immature intrafusal fiber and that these rudimentary spindles fail to progress beyond $\mathrm{E} 18$ and never develop into mature muscle spindles (Leu et al., 2003). To characterize the molecular development of muscle spindles in the ErbB2 $\Delta$ mutant, we used in situ hybridization analysis of $\mathrm{P} 1, \mathrm{P} 3$, and $\mathrm{P} 7$ animals with probes specific for the transcription factors Egr3 (Tourtellotte and Milbrandt, 1998) and Er81 (Arber et al., 2000), two molecular markers of muscle spindle differentiation that are first expressed at E15.5 and E18.5, respectively (Hippenmeyer et al., 2002)

Muscle spindle counts were done on TA muscles isolated from ErbB2 $\Delta$ mutant animals, as well as ErbB2 ${ }^{\text {FLOX/FLOX }}$ and ErbB2 ${ }^{\text {NULL/FLOX }}$ controls. At postnatal day 3 in the ErbB2 control animals, there were $31.0 \pm 1.9(n=5)$ muscle spindles per TA muscle, and these spindles were characterized by the strong expression of Egr3 in multiple intrafusal muscle fibers (Fig. $1 A$ ). In contrast, TA muscles in the ErbB2 $\Delta$ mutant expressed Egr3 weakly in only $9.6 \pm 3.8(n=5)$ rudimentary muscle spindles that comprised only a single Egr ${ }^{+}$myofiber (Fig. 1D). Conditional elimination of ErbB2 by $m y f 5^{C R E}$ therefore results in a 70\% loss in the total number of nascent muscle spindles as determined by the expression of Egr3. Moreover, because normal muscle spindles each contain up to four intrafusal fibers (Tourtellotte et al., 2001), there is a loss of $>90 \%$ of the total number of intrafusal fibers in the ErbB2 $\Delta$ mutant.

Additional in situ hybridization analysis with probes for Egr3 and Er81 on contiguous sections of TA muscle found no evidence of Er81 expression in any of the Egr $3^{+}$myofibers (Fig. $1 E$ ), in contrast to the ErbB2 controls in which Er81 is coexpressed in $\mathrm{Egr}^{+}{ }^{+}$spindles (Fig. $1 \mathrm{~B}$ ). In the TA muscle at P3, there is a near absence of Er81 ${ }^{+}$muscle spindles in the ErbB2 $\Delta$ mutant ( $0.6 \pm$ $0.2 ; n=5)$ compared with the ErbB2 $(27.5 \pm 1.9 ; n=4)$ controls (Fig. 1I). Similar results were obtained at postnatal days 1 and 7 (data not shown).

We also examined the expression of another molecular marker of muscle spindles, the neurotrophic factor NT3 that is initially expressed diffusely in embryonic muscle but becomes restricted to the intrafusal fibers late in embryogenesis (Copray and Brouwer, 1994; Wright et al., 1997). In situ analysis of NT3 expression revealed no detectable NT3 message at P3 in the ErbB2 $\Delta$ mutant (Fig. $1 F$ ) in contrast to the ErbB2 control (Fig. 1C).

These data demonstrate that, in the ErbB2 $\Delta$ mutants, there is widespread failure of muscle spindle induction, although the in- 

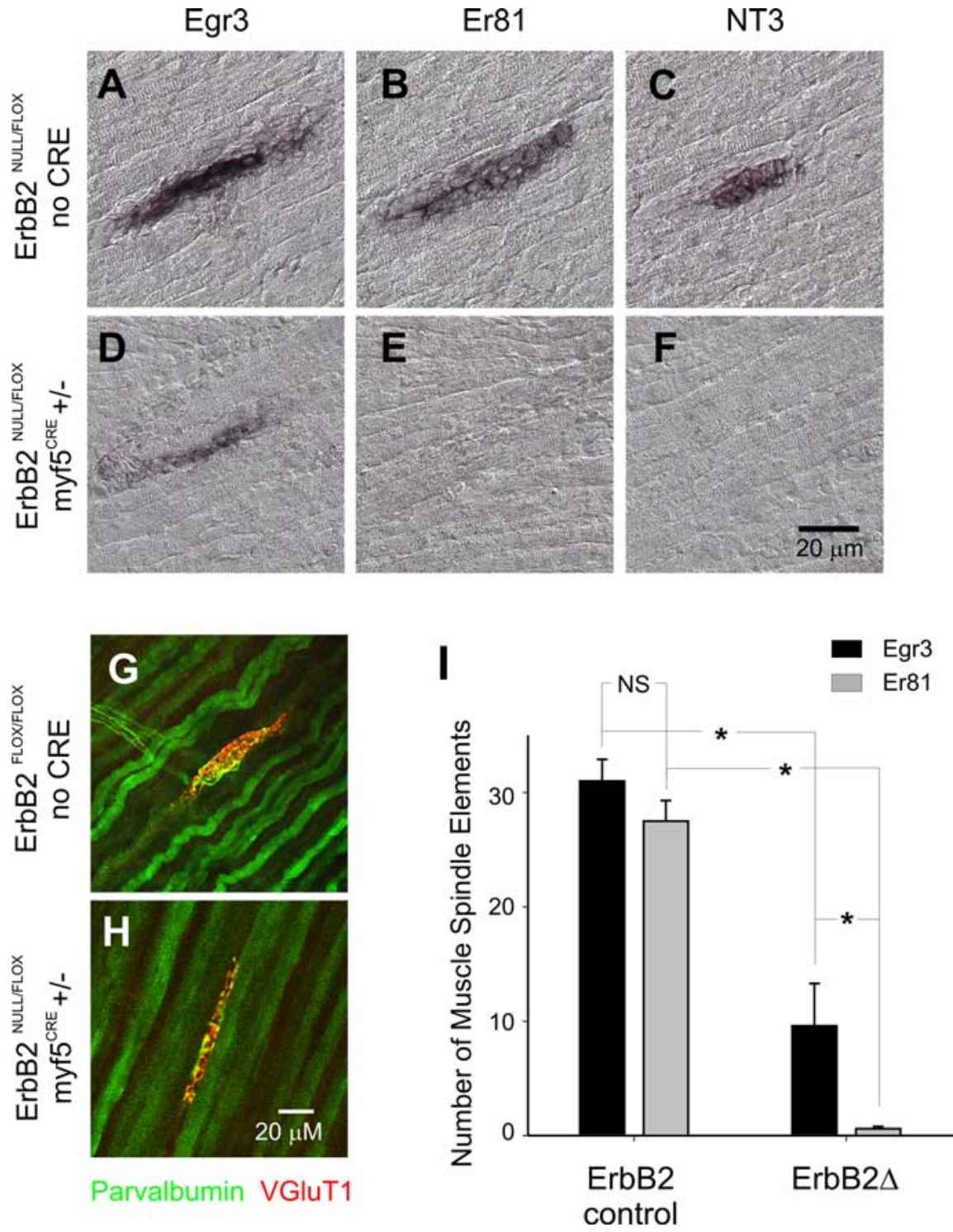

Figure 1. Late muscle spindle marker expression is decreased in ErbB2 $\Delta$ mutants. In situ hybridization analysis on serial sections of $\mathrm{P} 3$ tibialis anterior muscle from an ErbB2 $\Delta$ mutant $(\boldsymbol{D}-\boldsymbol{F})$ ErbB2 ${ }^{\mathrm{NULL} / F L 0 X}$ (no CRE) control $(\boldsymbol{A}-\boldsymbol{C}$ ) animal with probes for the transcription factors Egr3 $(\boldsymbol{A}, \boldsymbol{D}), \operatorname{Er} 81(\boldsymbol{B}, \boldsymbol{E})$, and neurotrophic factor NT3 $(\boldsymbol{C}, \boldsymbol{F})$ reveals low levels of Egr3 expression in a single myofiber of the mutant muscle spindle (D); Egr3 is robustly expressed in multiple intrafusal fibers in the control $(\boldsymbol{A})$. In situ analysis also demonstrates the complete absence of late spindle markers Er81 and NT3 in mutant muscle spindles $(\boldsymbol{E}, \boldsymbol{F})$ compared with the control ( $\boldsymbol{B}, \boldsymbol{C}$. Immunohistochemical analysis by whole-mount staining of $\mathrm{P} 4 \mathrm{gluteus}$ maximus muscle with antibodies against $\mathrm{PV}$

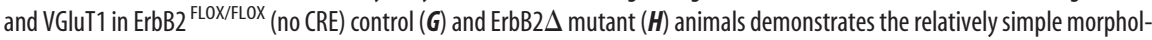
ogy of the muscle spindle afferent terminal around a single, rudimentary intrafusal fiber in the mutant. I, Quantitative in situ hybridization studies of the TA muscle of the ErbB2 $\Delta$ mutant demonstrate induction of Egr3 expression (black) in $~ 30 \%$ (9.6 \pm 3.8 SEM; $n=5$ ) of the number of spindles observed in ErbB2 (ErbB2 ${ }^{\text {NULL/FLOX }}$ or ErbB2 ${ }^{\text {FLOX/FLOX }}$ ) controls $(31.0 \pm 1.9 ; n=5)$. In contrast, no significant Er81 expression (gray) was observed in P3 mutant muscle. Statistical significance: * $p<0.001$, ANOVA (Fisher's least significant difference method); there was no statistically significant difference between the number of muscle spindles expressing Egr3 and Er81 in the ErbB2 controls.

hibition is not complete. In approximately one-third of the normal number of muscle spindles, induction initiates at E15, as demonstrated by the limited expression of Egr3, but is aborted before the expression of Er81 and NT3 at E18.

We next assessed the development of proprioceptive afferent terminals on muscle spindles in the ErbB2 $\Delta$ mutant by immunostaining with antibodies for the calcium binding protein $\mathrm{PV}$, a selective marker for proprioceptive sensory neurons (Honda, 1995; Arber et al., 2000), and VGluT1 that labels the proprioceptive afferent terminals on the muscle spindle (Wu et al., 2004).
Whole-mount immunostaining of the P4 gluteus maximus muscle was used to visualize the morphology of afferent terminals on the muscle spindle. Few of the rudimentary spindles remaining in the ErbB2 $\Delta$ mutant (Fig. $1 \mathrm{H}$ ) showed the characteristic, complex annulospiral afferent terminal normally found around the equatorial region of each intrafusal fiber (Fig. 1G). Moreover, the VGluT1 staining normally associated with the spindle afferent terminal was diminished around the ErbB2 $\Delta$ mutant muscle spindle compared with the $\operatorname{ErbB2} 2^{\text {FLOX/FLOX }}$ and wild-type controls (Fig. 1G,H) (WT data not shown). In comparison, the morphology of the Golgi tendon organ, a muscle mechanoreceptor innervated by class $\mathrm{I}_{\mathrm{B}}$ afferents, was unaffected by the elimination of muscle ErbB2 (data not shown), consistent with the selective elimination of muscle spindles in animals lacking neuronal Nrg1 (Hippenmeyer et al., 2002).

The widespread failure of muscle spindle differentiation in the ErbB $2 \Delta$ mutant had no obvious effect on muscle spindle afferent survival, as expected from the analysis of the neuronal Nrg1 mutant (Hippenmeyer et al., 2002), and similar mutants in which ErbB2 expression is eliminated from developing muscle (Leu et al., 2003). Anterograde labeling of sensory projections to the spinal cord from the L5 dorsal root (Fig. 2A,D) together with parvalbumin immunostaining (Fig. $2 B, E$ ) demonstrated that muscle spindle afferents projected to the ventral spinal cord in their characteristic pattern (P4 through P7).

Immunostaining for VGluT1, which is enriched in the central axonal terminals of postnatal DRG sensory neurons (Oliveira et al., 2003), was also observed throughout the mutant spinal cord in a pattern indistinguishable from the control animals (Fig. 2C,F). To determine whether proprioceptive afferents make direct contacts on motor neurons in the mutant animals, we visualized presynaptic afferent terminals by the colocalization of Texas Red Dextran (from anterograde L5 dorsal root fills), PV, and VGluT1 (Fig. 2G-I, $J-L$, respectively) on motor neurons labeled retrogradely from the ventral root. By this method, we observed triplelabeled L5 afferent terminals in contact with L5 motor neurons in the ErbB2 $\Delta$ mutants throughout the neonatal period, to as late as P8.

We extended our histochemical analysis of these mutants to P21 to establish whether the afferent synaptic contacts we detected at $\mathrm{P} 6$ persisted as the animal matured. For this purpose, we counted the number of VGluT1 ${ }^{+}$contacts on the cell bodies of large-diameter motor neurons in the L4 lateral motor column in the mutant and wild-type controls (Fig. 3). In this analysis, three- 
dimensional reconstructions of motor neuron somata were first obtained from z-series of confocal optical images, from which estimates of individual cell surface area were calculated to determine approximate VGluT $1{ }^{+}$contact density per soma. Small-diameter motor neurons $<10,000$ $\mu \mathrm{m}^{2}$ were excluded from analysis, and there was no significant difference between the average surface area of mutant $\left(13,263 \pm 372 \mu \mathrm{m}^{2} ; n=20\right)$ and control $\left(12,188 \pm 433 \mu \mathrm{m}^{2} ; n=9\right)$ motor neurons ( $p=0.1, t$ test). On average, there were $18.5 \pm 1.15(n=20) \mathrm{VGluT}^{+}{ }^{+}$contacts on motor neurons in the ErbB2 $\Delta$ mutant compared with $26 \pm 2.35(n=9)$ in the control, corresponding to a contact density of $0.14 \pm 0.008$ and $0.20 \pm 0.025$ contacts per $100 \mu \mathrm{m}^{2}$ surface area on mutant and control motor neurons, respectively. Although the reduction in the number of VGluT1 did not reach significance, the density of VGluT1 contacts was reduced by $\sim 30 \%$ in the ErbB2 $\Delta$ mutant (Fig. 3C,E). These data demonstrate that, in the absence of normal spindle development, there is only a modest reduction in the normal density of $\mathrm{VGluT}^{+}$contacts on motor neurons, which led us to examine whether these anatomical contacts between muscle afferents and motor neurons are functional in the ErbB2 $\Delta$ muscle spindle mutants.

Electrophysiological characterization of monosynaptic connections between muscle-identified primary afferents and motor neurons

To record afferent-evoked responses from muscle-identified motor neurons, we visually targeted motor neurons prelabeled 2-3 d earlier by retrograde transport of a tracer injected into the TA or the LG muscles. During the physiological experiment, the recorded motor neuron was injected with a different dye. After processing the tissue for immunohistochemistry, we confirmed that the recorded cells were labeled with both the intracellular and the retrogradely transported marker (Fig. 4 (also see Fig. $6 A, B$, bottom left insets). By prelabeling TA and LG motor pools in vivo, we were able to cut the ventral roots and thereby avoid using antidromic stimulation of a muscle nerve to determine the identity of motor neurons (Seebach and Ziskind-Conhaim, 1994; Mears and Frank 1997). Muscle-nerve stimulation results in an antidromic action potential in the homonymous motor neuron and the disynaptic recurrent IPSP (Mentis et al., 2005), which would have complicated detection of the afferent-evoked EPSPs. Moreover, monosynaptic inputs from other motor neurons (Mentis et al., 2005; Nishimaru et al., 2005) would have been impossible to distinguish from monosynaptic EPSPs generated by stimulation of muscle afferents. Cutting the ventral roots made it possible to restrict the effects of muscle nerve stimulation to those attributable solely to the activation of afferents.
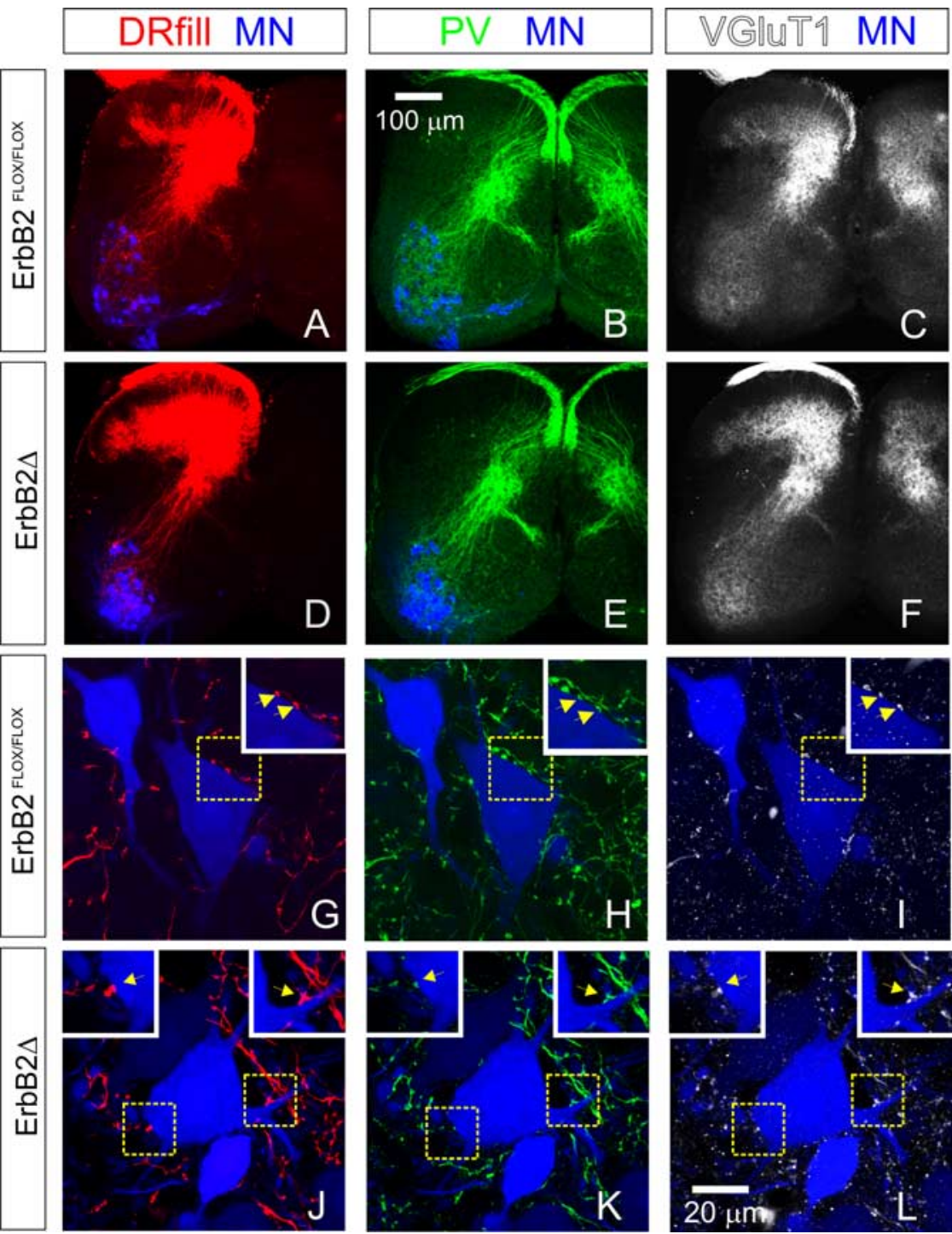

Figure 2. The gross pattern of central projections is normal in ErbB2 $\Delta$ mutant mice. $\boldsymbol{A}-\boldsymbol{F}$, The pattern of central projections of

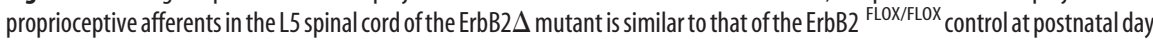
6. The images are z-stack projections of $\sim 10$ scans of $2 \mu \mathrm{m}$ thickness. $\boldsymbol{A}, \boldsymbol{B}, \boldsymbol{D}, \boldsymbol{E}$, The $L 5$ dorsal root was filled with Texas Red distribution of immunostaining for the VGluT1 is normal in the ErbB2 $\Delta$ mutants. $\mathbf{G}-\boldsymbol{L}$, Proprioceptive afferent terminals, triplelabeled with Texas Red Dextran, PV, and VGlut1on L5 motor neurons in the ErbB2 ${ }^{\text {FLOX/FLOX }}$ control $(\mathbf{G}-\boldsymbol{I})$ and ErbB2 $\Delta(\boldsymbol{J}-\boldsymbol{L})$ mutant. The insets in the top corners are enlargements of the yellow boxes; synaptic contacts are indicated by the yellow arrows.

We examined the synaptic potentials evoked by afferent stimulation in nine motor neurons in nine wild-type animals (P4 and P5) and in eight motor neurons in eight ErbB2 $\Delta$ mutant animals (P4 and P5). To establish that the afferent-evoked synaptic potentials recorded in wild-type and mutant animals were monosynaptic, we compared the timing of the afferent-evoked extracellular field potentials recorded within the motor nucleus with the onset of the homonymous ventral root potential and the intracellularly recorded afferent-evoked synaptic potential (Fig. 5). As has been shown previously in the mouse (Li and Burke, 2001), the afferent-evoked field potential comprises two main components. The first is the terminal potential representing orthodromic invasion of muscle afferent fibers within the motor nucleus (Fig. 5A, red trace, field potential) (Lev-Tov and Pinco, 1992; Li and Burke, 2001), and the second component is the synaptic field potential. The synaptic field potential was identi- 


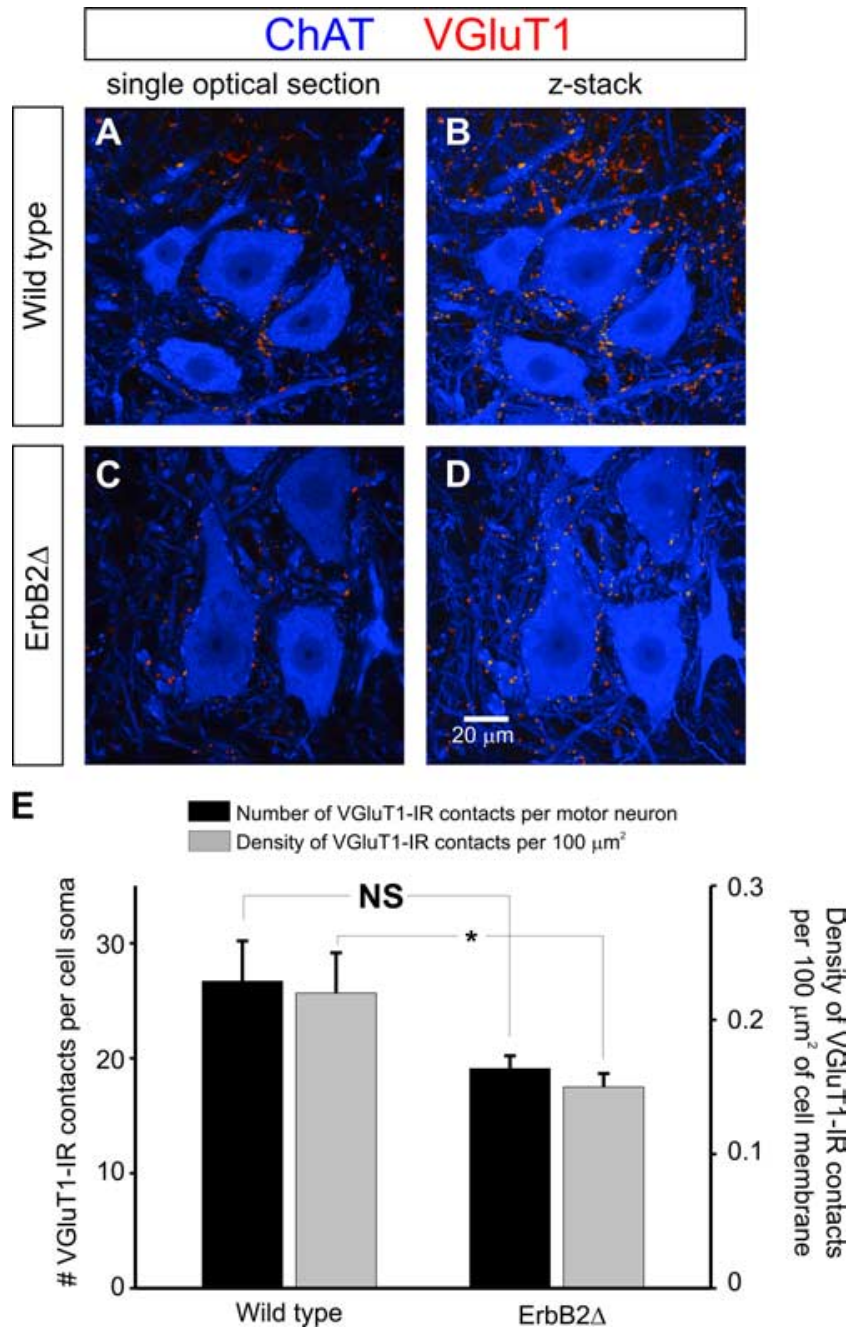

Figure 3. At P21, VGluT1 ${ }^{+}$contacts on motor neurons are reduced in the ErbB2 $\Delta$ mice. $\boldsymbol{A}-\boldsymbol{D}$, Single optical plane (step, $0.6 \mu \mathrm{m})(\boldsymbol{A}, \boldsymbol{C})$ and projections of $\sim 10$ optical planes (single optical thickness, $0.6 \mu \mathrm{m})(\boldsymbol{B}, \boldsymbol{D})$ of two $\mathrm{L} 4$ motor neurons from a $\mathrm{P} 21$ wild-type control $(\boldsymbol{A}, \boldsymbol{B})$ and P21 mutant $(\boldsymbol{C}, \boldsymbol{D})$. Motor neurons were identified by ChAT staining (blue); VGluT1 contacts are shown in red. $\boldsymbol{E}$, Quantitative analysis of VGluT1 ${ }^{+}$contacts per motor neuron soma and corresponding densities in the mutant and wild-type control demonstrate no significant (NS) loss of VGluT1 contacts on motor neuron somata in the mutant and a modest but significant $\left(p<0.05\right.$ ) decrement of $\sim 30 \%$ in the density of VGluT1 ${ }^{+}$terminals. No significant difference was observed in the size of motor neurons analyzed in the mutant versus control.

fied by comparing the field potentials in the presence and absence of low calcium to block synaptic transmission (Fig. 5A). These recordings revealed that the onset of the synaptic field potential coincided with the onset of the electrotonically recorded ventral root potential (Fig. $5 A$, right panel, double-headed arrow). In three experiments, this allowed us to approximate the synaptic delay as the time between the onsets of the terminal and synaptic field potentials (mean of $0.85 \mathrm{~ms}$ ). However, this is probably an overestimate of the delay because extracellular recordings may not be sensitive enough to detect the earliest synaptic events in motor neurons. In accord with this interpretation, we found that the latency of the intracellularly recorded EPSP generated by stimulation of the homonymous muscle nerve was $0.9 \pm 0.4 \mathrm{~ms}$ (range, -1.9 to $0.6 \mathrm{~ms}$ ) less than that of the onset of the ventral root potential (Fig. 5C,D), consistent with the monosynaptic origin of the earliest part of the EPSP.

As an additional control, because ErbB2 is also involved in the differentiation and growth of Schwann cells that could be affected in the ErbB2 $\Delta$ mutant (for review, see Britsch, 2007), we measured the conduction velocity of the primary afferents activated from the peripheral nerves and found that, in wild-type mice $(n=4)$, the average conduction velocity from the nerve to the DRG was $1.45 \pm 0.06 \mathrm{~m} / \mathrm{s}$ and from the nerve to the recorded motor neuron was $1.26 \pm 0.04 \mathrm{~m} / \mathrm{s}(n=5)$. Similarly for the ErbB2 $\Delta$ mice, the average conduction velocity from the muscle nerve to the DRG was $1.82 \pm 0.14 \mathrm{~m} / \mathrm{s}(n=4)$ and from the muscle nerve to motor neuron was $1.44 \pm 0.19 \mathrm{~m} / \mathrm{s}(n=5)$ (Fig. $5 E)$. There was no statistical difference between wild-type and ErbB2 $\Delta$ mice ( $t$ test, $p>0.50$ ).

\section{Monosynaptic inputs from muscle spindle afferents to motor neurons are functional but reduced in amplitude in the ErbB2 $\Delta$ mutant}

Figure 6 illustrates the method used to determine the pool identity of two LG motor neurons from a wild-type (Fig. 6A) and an ErbB2 $\Delta$ (Fig. $6 B$ ) mouse. In each case, we used visual targeting to record from cells labeled with the retrograde tracer $\mathrm{CTb}-647$ and then injected the cell with Alexa 543 hydrazide (Fig. 6A, $B$, red cells in main panel). After fixing the spinal cords and processing the tissue for immunohistochemistry, we confirmed that both motor neurons were double labeled with the intracellular and the retrograde dyes (Fig. 6A, $B$, yellow arrows in the small insets). This approach also allowed us to determine whether VGluT1 terminals were also present on the dendrites of the recorded motor neurons. Although we demonstrated the presence of VGluT ${ }^{+}$terminals on motor neuron somata in both wild-type and ErbB2 $\Delta$ animals, in adult animals it is known that most of the muscle afferent input onto motor neurons is on the dendrites (Burke and Glenn, 1996). As shown in the large insets in Figure 6, we confirmed the presence of VGluT1 ${ }^{+}$terminals on motor neuron dendrites for both wild-type and $\operatorname{ErbB} 2 \Delta$ animals.

We measured the amplitude of the monosynaptic response in both wild-type and ErbB $2 \Delta$ mice 3 ms after the onset of the EPSP. We chose this time based on two observations. First, the difference in the latency of the homonymous and antagonist EPSPs was 5-6 ms for wild-type and ErbB $2 \Delta$ animals (see below). Second, we sometimes observed an inflection on the rising phase of the EPSP (supplemental Fig. $1 A$, available at www.jneurosci.org as supplemental material) that occurred at $4.3 \pm 0.4 \mathrm{~ms}$ after the EPSP onset for three wild-type motor neurons (one LG and two TA) and $4.5 \pm 0.4 \mathrm{~ms}$ for three ErbB $2 \Delta$ motor neurons (two LG and one TA; $2 \times$ or $5 \times \mathrm{T}$ stimulation). It seems reasonable to suppose that the inflection coincides with activation of a disynaptic component to the afferent-evoked EPSP. Consistent with this interpretation, we found that the delayed component exhibited greater synaptic depression during a stimulus train of $10 \mathrm{~Hz}$ than did the initial component of the EPSP. Because of the rapid depression that occurs during such a stimulus train (supplemental Fig. 4, available at www.jneurosci.org as supplemental material), we quantified the depression by expressing the amplitude of the second response in the stimulus train as a percentage of the first response. In two wild-type motor neurons, the initial component of the response (measured at $3 \mathrm{~ms}$ after the EPSP onset) fell to $70.5 \%$ of the amplitude of the first response in the stimulus train. The later peak (measured at 6-6.5 ms after the EPSP onset) fell to $54 \%$ of the amplitude of the first response in the stimulus train. Similarly for three ErbB $2 \Delta$ motor neurons, the early response $(3 \mathrm{~ms})$ fell to $63 \pm 3.6 \%$ of the amplitude of the first response in the train, whereas the later response $(6-6.5 \mathrm{~ms})$ fell to $48.3 \pm 2.4 \%$ ( $t$ test, $p<0.05$ ). The latencies of the inflections $(4.3$ $\mathrm{ms}$ wild type; $4.5 \mathrm{~ms}$ ErbB2 $\Delta$ ) are similar to those of the Ia inhib- 
itory disynaptic IPSP in neonatal (P7) mouse motor neurons (Wang et al., 2008). These authors showed that the onset of the reciprocal Ia IPSP occurred $3.5 \mathrm{~ms}$ after the onset of the homonymous EPSP in P7 mouse motor neurons.

Our method for measuring the amplitude of the monosynaptic component of the afferent-evoked potential differs from that used by Frank and his coworkers (for a description of the method, see Mears and Frank, 1997) who used a "model” PSP to measure the amplitude of the monosynaptic potentials. In supplemental Figure $1 B$ (available at www.jneurosci.org as supplemental material), we compare the two methods and show that they produce very similar estimates for the amplitude of the monosynaptic EPSP, with the latency method used here producing a slightly lower estimate of the amplitude than the "model" method.

We found that the amplitude of monosynaptic potentials was reduced by $\sim 80 \%$ in the ErbB2 $\Delta$ animals compared with wild-type animals (Fig. 6C,D). The average amplitude of five homonymous EPSPs in wild type animals (three LG and two TA motor neurons) was $5.5 \pm 2.4 \mathrm{mV}$, and for six cells in the ErbB2 $\Delta$ animals (four LG and two TA motor neurons), it was $1.2 \pm$ $0.5 \mathrm{mV}$. The difference was statistically significant ( $p<0.05$, Mann-Whitney test). As with the intracellular EPSPs, the measurements of extracellularly recorded ventral root potentials were made at $3 \mathrm{~ms}$ after the onset of the potential (or at the peak if it occurred within $3 \mathrm{~ms}$ of the stimulus) to ensure that only the monosynaptic component was measured. Stimulation of the dorsal root resulted in a ventral root potential whose amplitude was $5.0 \pm 0.7 \mathrm{mV}$ for the wild-type and $1.3 \pm 0.2 \mathrm{mV}$ in the ErbB2 $\Delta$ mice. This difference in the amplitude of the afferent-evoked ventral root potentials was also statistically significant $(p<0.05$, MannWhitney test). Similarly, the ventral root potentials generated in response to stimulation of the muscle nerves were also significantly smaller in the ErbB2 $\Delta$ animals $(p<0.05)$. In the wild-type animals, stimulation of the LG or TA nerves generated average ventral root potentials of $0.89 \pm 0.28$ or $0.94 \pm 0.19 \mathrm{mV}$, respectively. For the ErbB2 $\Delta$ animals, these values were $0.19 \pm 0.03$ and $0.14 \pm 0.07 \mathrm{mV}$, respectively, a reduction of $80-85 \%$.

The differences in the amplitude of the EPSPs in wild-type and ErbB2 $\Delta$ mice are not attributable to differences in the input resistance of the motor neurons. The input resistance measured in four LG wild-type motor neurons was $37.1 \pm 7.3 \mathrm{M} \Omega$, which was not statistically different $(t$ test) from the values measured in five LG ErbB $2 \Delta$ motor neurons $(28.1 \pm 9.5 \mathrm{M} \Omega)$. In addition, five TA wild-type motor neurons had a mean of input resistance of $36.7 \pm 7.3 \mathrm{M} \Omega$, similar to the value obtained from three TA ErbB2 $\Delta$ motor neurons $(38.8 \pm 9.5 \mathrm{M} \Omega$ ).

The reduction in the amplitude of the afferent-evoked synap-
Cell attached Whole Cell
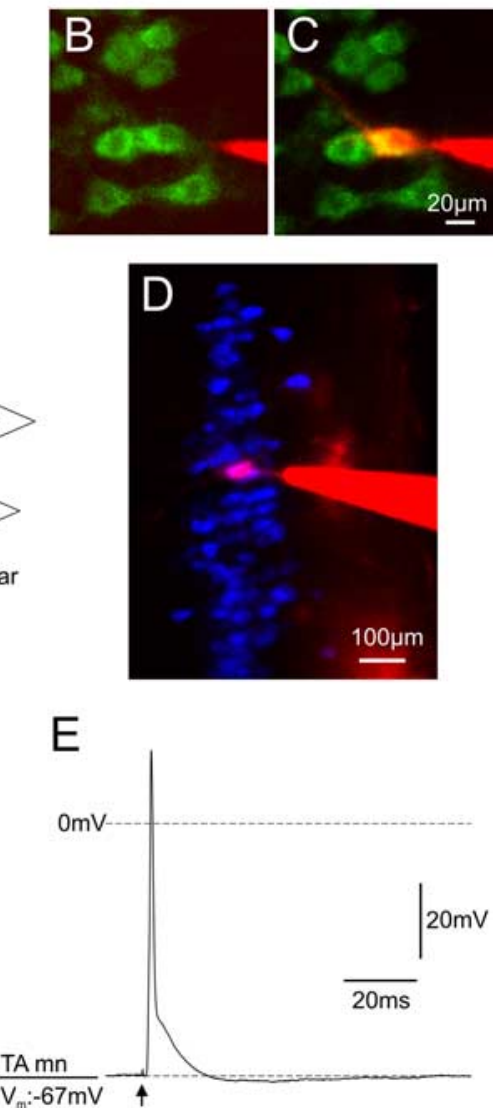

Figure 4. The isolated spinal cord preparation and identification of motor neurons. $A$, Shows a drawing of the lumbar spinal cord dissected together with the sciatic nerve and its major peripheral nerves. Recording and stimulating electrodes were applied and L5 lumbar ventral roots (vr-L4 and vr-L5), en passant on the L4 and L5 dorsal root ganglia (DRG-L4 and DRG-L5), and

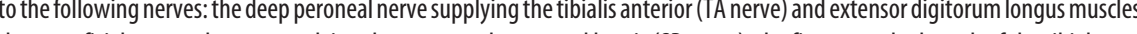
superficial peroneal nerve supplying the peroneus longus and brevis (SP nerve), the first muscular branch of the tibial nerve nerve). In some experiments, an extracellular glass electrode was used to record afferent-evoked field potentials. $B, C_{c}$ together with a microelectrode filled with Alexa 543 hydrazide (red) cell attached to a TA motor neuron $(\boldsymbol{B})$ and after rupture of the

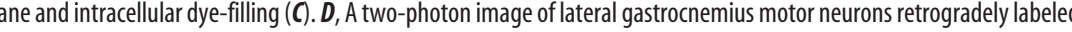
whole-cell recording (pink because of red and blue colocalization). $\boldsymbol{E}$, an antidromically evoked action potential recorded from a wild-type $L G$ motor neuron and evoked by stimulation of the $L 4$ ventral root.

tic potentials in the ErbB2 $\Delta$ animals cannot be explained by an inability to stimulate afferent axons in the peripheral nerve, either because their electrical thresholds were too high or because they had retracted within the muscle nerve beyond the point of stimulation. This is because the monosynaptic EPSPs evoked by dorsal root stimulation (which would have stimulated all afferent fibers) were reduced by a similar amount (26\% of wild-type amplitude) to those evoked by muscle nerve stimulation (22\% of wild-type amplitude).

The effects of conditional elimination of spindle-derived NT3 on the amplitude of monosynaptic ventral root potentials evoked by muscle afferent stimulation

As described above, the elimination of ErbB2 expression in muscle precursors results in the arrest of muscle spindle development before E18 and a variety of molecular and morphological defects in spindle development. One of these is the loss of NT3 expression from muscle spindles (Fig. 1C,F), which previous studies 


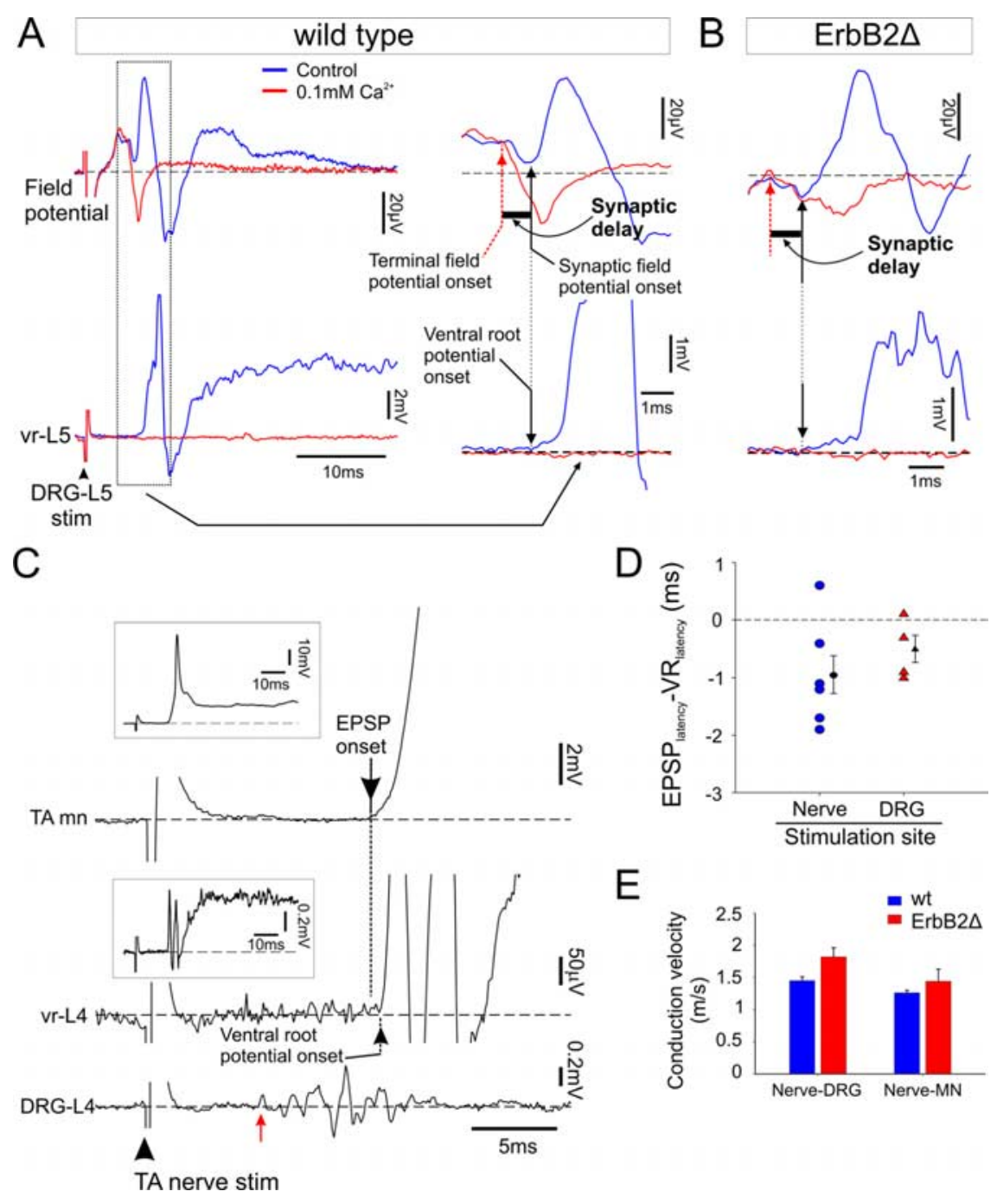

Figure 5. Temporal relationships between afferent-evoked field potentials, ventral root potentials, and intracellularly recorded EPSPs from muscle-identified motor neurons. $A$, Simultaneous recording of the extracellular field potentials (Field potential; top) recorded within the motor nucleus at the L4/5 border and the $L 5$ ventral root potential (vr-L5; bottom) in control aCSF (blue traces) and in a low $(0.1 \mathrm{~mm}$ ) calcium aCSF (red traces) to block chemical synaptic transmission. The recordings were made in a wild-type $P 5$ mouse. The traces on the right are shown at an expanded time base indicated by the dotted box on the left panel. The field potential recorded in low-calcium aCSF is the terminal potential (labeled as "Terminal field potential onset" and is marked by red arrowed dotted line) and is generated by action potentials invading primary afferent arbors within the motor nucleus. The synaptic field potential starts where the field potential recorded in normal aCSF deviates from the low calcium trace (labeled as "Synaptic field potential onset" and marked by black arrow). Note that the onset of the synaptic field potential coincides with the onset of the electrotonically recorded ventral root potential (labeled as "Ventral root potential onset" and marked by dotted line with arrowhead). The field potential traces are averages from 250 individual responses ( $10 \mathrm{~Hz}$ at supramaximal stimulus intensity) so that the synaptic field potential amplitude is depressed. The ventral root potential is not averaged. The response to the first stimulus is shown for each condition. The synaptic delay is approximated as the time from the onset of the terminal field (red arrow) to the onset of the synaptic field (black arrow). $\boldsymbol{B}$, Field potential and ventral root potentials recorded in a P5 ErbB2 $\Delta$ preparation. The color code is the same as in $\boldsymbol{A}$. Note that there was no difference in the synaptic delay between wild-type and ErbB2 $\Delta$ mice. C, Comparison of the timing of the synaptic potential recorded in a tibialis anterior motor neuron (TA $\mathrm{mn}$ ) evoked by stimulation of the TA muscle nerve $(5 \times \mathrm{T}$ at $0.1 \mathrm{~Hz})$ with the simultaneously recorded $\mathrm{L} 4$ ventral root potential (vr-L4) and the afferent volley recorded from the L4 dorsal root ganglion (DRG-L4) in a P4 wild-type mouse. Each recording has been averaged from five traces. Insets above the top (TA $\mathrm{mn}$ ) and middle (vr-L4) traces show the responses at a slower timescale. Note that, in this cell, the onset of the homonymous synaptic potential precedes the onset of the ventral root potential consistent with its origin monosynaptically. The red arrow under the DRG-L4 trace identifies the timing of the earliest part of the volley that was used to calculate the conduction velocity of the primary afferent axons. $D$, Graph showing the difference in time between the onset of intracellularly recorded EPSPs from wild type TA and LG motor neurons and the ventral potentials generated by stimulation of the homonymous muscle nerve or the DRG (DRG-L4 for TA and DRG-L5 for LG). The stimulation intensity was $5 \times$ T at 0.1 $\mathrm{Hz}$. The onset times were calculated from averages of three to five sweeps. The black symbols show the average and SEM for the data. $\boldsymbol{E}$, Graph showing the conduction velocities of the primary afferents calculated from the latency from stimulation of the peripheral nerve to the volley recorded in the DRG (Nerve-DRG) or the latencies from stimulation of the peripheral nerve to the onset of the EPSP recorded in the motor neuron (Nerve-MN). There were no statistical differences in the conduction velocities between the wild type ( $n=5$; blue) and $\operatorname{ErbB} 2 \Delta$ ( $n=5$; red) mice ( $t$ test; $p=0.47$ for Nerve-DRG; $p=0.38$ for Nerve-MN). suggest accounts for the loss of the monosynaptic sensorimotor responses observed in the Erg3 knock-out mouse in which muscle spindle development is abnormal, and spindles do not express NT3 (Chen et al., 2002). To determine genetically whether the attenuation of the afferentevoked monosynaptic EPSPs in the ErbB2 $\Delta$ animals was attributable to the loss of spindle-derived NT3 rather than some other abnormality of spindle development, we generated a novel Egr ${ }^{C R E}$ allele and used it in combination with a floxed allele of NT3 (Bates et al., 1999) to selectively eliminate the expression of NT3 from the intrafusal fibers of muscle spindles. The Egr $3^{C R E}$ allele was designed to preserve the expression of endogenous Egr3 by placing an IRES-CRE expression sequence downstream of the last coding exon of the Egr3 gene (supplemental Fig. 2, available at www.jneurosci.org as supplemental material). Animals homozygous for both the $N T 3^{\text {FLOX }}$ and Egr $3^{\mathrm{CRE}}$ alleles were generated in a predominantly C57BL/6J genetic background, and serial sections of neonatal muscle were analyzed by in situ hybridization for the expression of the early spindle markers Egr3, Er81, and NT3. At P1, spindles of the wild-type (data not shown) and NT3 ${ }^{\text {FLOX/FLOX }}$ (no CRE) control animals expressed each of these late spindle markers; however, in the NT3 ${ }^{\text {FLOX/FLOX }} /$ Egr $^{\text {CRE/CRE }}$ mutants (referred to as NT3 $\Delta$ in the subsequent text and figures), NT3 was selectively absent (Fig. 7). Similar analysis at P3 demonstrates that NT3 expression is not simply delayed (data not shown). The NT3 $\Delta$ mutants demonstrated the same ataxic gait and flexure posturing as the ErbB2 $\Delta$ mutants, consistent with a deficit in proprioceptive signaling, although the motor phenotype observed was delayed in onset and less pronounced than that observed in the ErbB2 $\Delta$ animals.

We compared the amplitude of ventral root potentials at P5 in NT3 $\Delta$ animals and wild-type controls, and surprisingly the amplitude of the monosynaptic response was normal in NT3 $\Delta$ mutants at P5, as illustrated in Figure $7 G$. However, at P14, by which age the NT3 $3^{\text {FLOX/FLOX }} /$ Egr3 $^{\text {CRE/CRE }}$ animals develop a proprioceptive phenotype, the amplitude of the potentials was significantly reduced (Fig. 7I). At P5, the average amplitude of the potentials measured was $8.3 \pm 1.7 \mathrm{mV}$ for the NT3 $\Delta$ animals $(n=3)$ and $6.4 \pm 1.1 \mathrm{mV}$ for the controls; none of these values differed statistically ( $p=0.10$, ANOVA). In contrast, at P15, the amplitude of the potentials measured was $0.35 \pm 0.03 \mathrm{mV}$ for the 

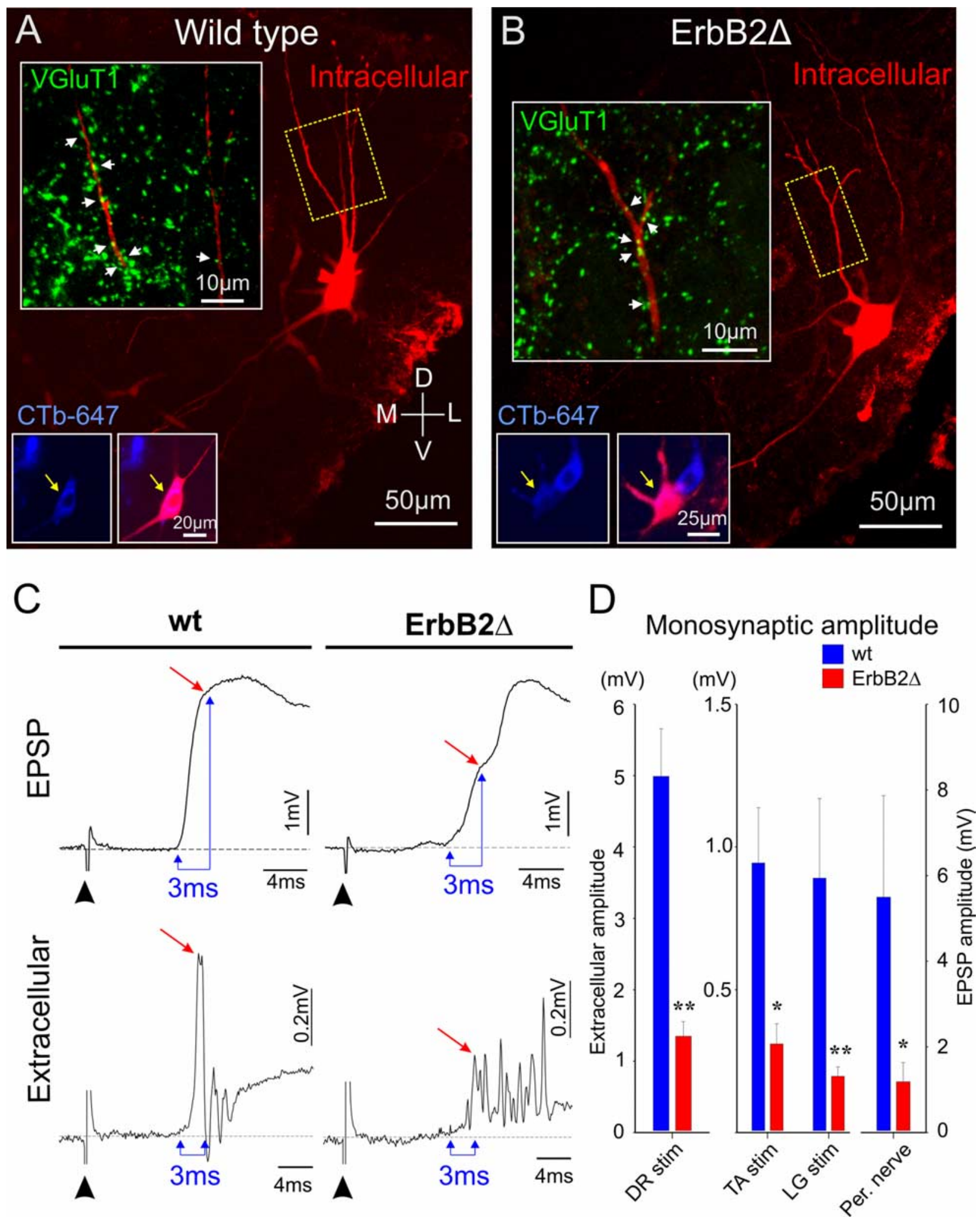

Figure 6. Monosynaptic connections between primary afferents and motor neurons are preserved but reduced in strength in ErbB2 $\Delta$ mice. $A, B$, Images of a wild-type $(A)$ and an ErbB2 $\Delta(B) L G$ motorneuron recorded from and subsequently filled with Alexa 543 hydrazide (shown in red). The images arez-stack projections of either 40 ( $A$ ) or $44(B)$ scans of $1.9 \mu$ m optical thickness. The identification of the cell as an $L G$ motor neuron was established by double labeling with the retrograde tracer $(T b-647$ (blue) as illustrated in the two insets on the bottom left of each image. Note that the double-labeled cells identified by the arrows are pink (blue and red), indicating colocalization, whereas the dye-filled cells shown in the main part of the image are red. Immunohistochemistry against VGluT1 revealed several VGluT1 ${ }^{+}$terminals apposed to the motor neuron dendrites. These can be seen in the large insets in both figures (white arrows), which show an expanded region off the dendrites indicated by the dotted yellowbox. Identification of VGluT $1{ }^{+}$terminal appositions was made on individual optical sections. Each image is a z-stack projection of 18 sections each $0.92 \mu \mathrm{m}$ thick. D, Dorsal; V, ventral; L, lateral; M, medial. C, Intracellularly recorded EPSPs together with simultaneously recorded ventral root potential in response to stimulation of the $L \mathrm{G}$ nerve $(5 \times \mathrm{T}$, average of 5 stimuli at $0.1 \mathrm{~Hz})$ in wild-type and ErbB2 $\Delta$ mice (P5). The amplitude of the monosynaptic response was measured for all records at $3 \mathrm{~ms}$ (indicated) after the onset of the EPSP or the ventral root potential (see Results). The black arrowheads indicate the stimulus artifact. Note that both the EPSP and the monosynaptic component of the ventral root potential are smaller in the ErbB2 $\Delta$ motor neurons compared with WT (indicated by the red arrows). $\boldsymbol{D}$, Bar graphs showing the (Figure legend continues.) 


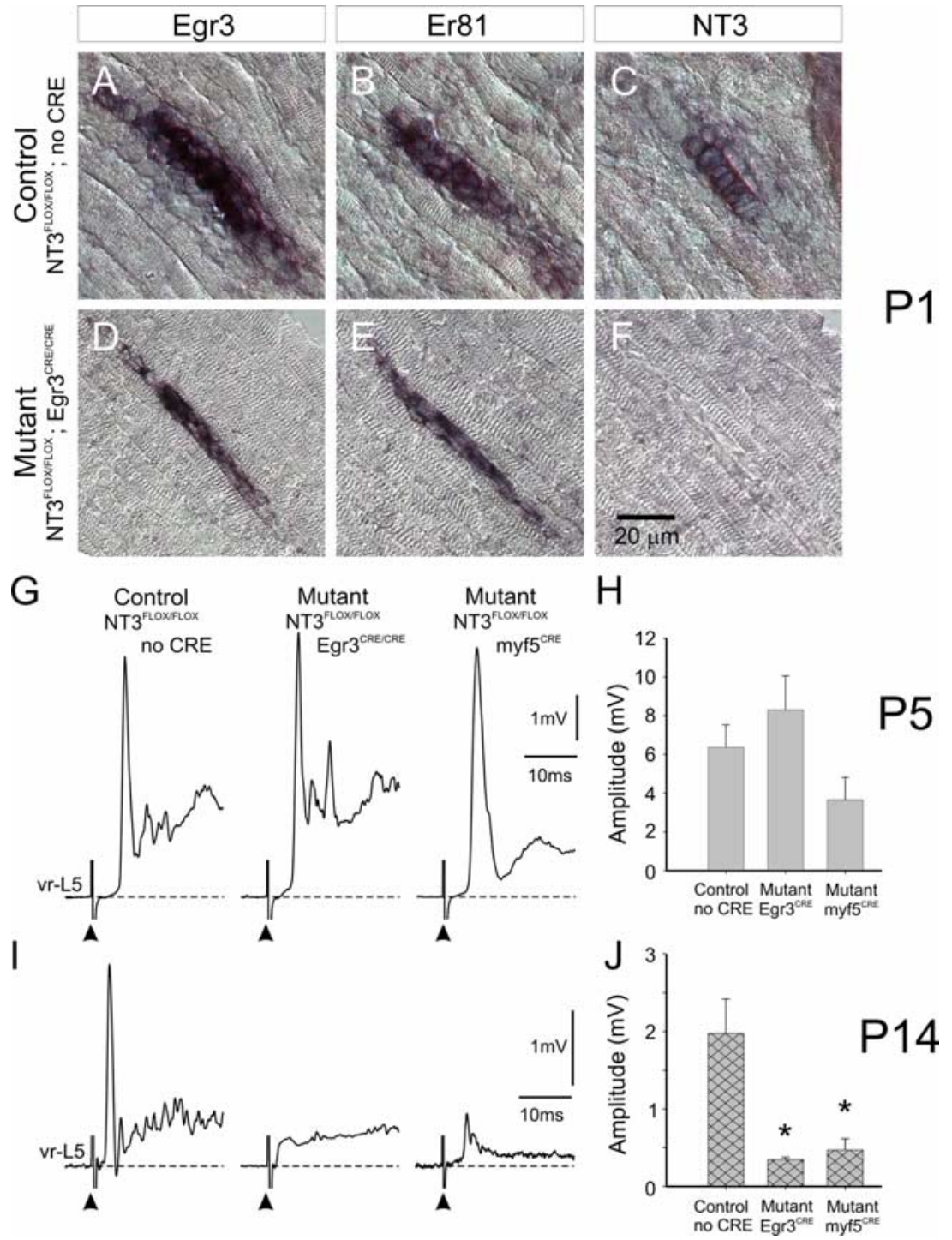

Figure 7. Selective elimination of NT3 expression in NT3 $\Delta$ mutants does not result in reduced afferent-evoked monosynaptic ventral root potentials at $P 5$. In situ hybridization analysis of serial sections of $P 1(\boldsymbol{A}-\boldsymbol{F})$ hindlimb from NT3 ${ }^{\text {FLOX/FLOX } / E g r 3} 3^{\text {CRE/CRE }}$

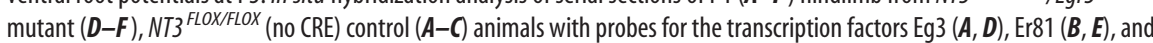
NT3 $(\boldsymbol{C}, \boldsymbol{F})$ reveals selective elimination of NT3 from mutant muscle spindles. $\boldsymbol{G}$, Averaged extracellular recordings from the L5 ventral root after stimulation of the $L 5$ dorsal root at $5 \times T$ from three groups of animals ( 1 control and 2 mutants) at P5. The frequency of stimulation was $0.1 \mathrm{~Hz}$. Note that the amplitude of the monosynaptic component the ventral root potential (arrows) did not differ significantly between the controls (NT3 ${ }^{\text {FLOX/FLOX }}$; no Cre) and either of the two mutants (NT3 ${ }^{\text {FLOX/FLOX }} /$ Egr3 $3^{\text {CRE/CRE }}$ or

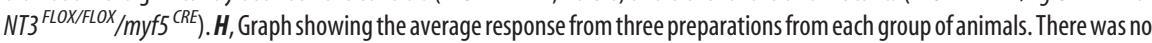
significant difference between the groups ( $p=0.10$, ANOVA). $\boldsymbol{I}$, Recordings from the same classes of animal as in $\mathbf{G}$ but at P14. Note that the monosynaptic reflex was greatly reduced in the mutants compared with the controls.J, Graph showing the averaged amplitude of the monosynaptic response from three animals per group. There was a significant reduction in the monosynaptic reflex for both mutants compared with that of the control group $\left({ }^{*} p<0.01\right.$, ANOVA Fisher's test).

NT3 $\Delta$ animals $(n=3)$ and $1.98 \pm 0.44 \mathrm{mV}$ for the controls $(n=$ 3 ), which represents a reduction of $>80 \%$.

Because Egr $3{ }^{\mathrm{CRE}}$ and NT3 are also expressed in Schwann cells and the peripheral vasculature (supplemental Fig. $2 C, D$, available

(Figure legend continued.) average amplitude of the ventral root potentials (left bars) and the intracellularly recorded EPSPS (right bars) in wild-type (blue) and ErbB2 $\Delta$ (red) animals in response to stimulation of the sites indicated below the plot. DR, Dorsal root; TA, tibialis anterior nerve; $L G$, lateral gastrocnemius nerve. The average intracellular response of the monosynaptic EPSP is shown for five wild-type (blue) and six ErbB2 $\Delta$ (red) $L G$ and TA motor neurons (per, peripheral nerve). Statistical significance: ${ }^{*} p<0.05,{ }^{* *} p<0.025, t$ test. at www.jneurosci.org as supplemental material) (Scarisbrick et al., 1993; Jessen and Mirsky, 2005), the effect on monosynaptic potentials we observe at P14 in the NT3A mice could be attributable to the loss of NT3 from these other, nonspindle sources. To address this possibility, we compared the amplitude of ventral root potentials at P5 and P14 in NT3 $\Delta$ animals to those in which NT3 was selectively eliminated from all (intrafusal and extrafusal) muscle fibers using the same myf5 ${ }^{\mathrm{CRE}}$ allele used in the generation of the ErbB2 $\Delta$ mutants. As illustrated in Figure 7, $G$ and $H$, the amplitude of the monosynaptic response was also normal

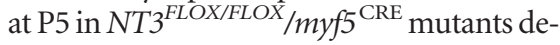
spite the absence of NT3 expression in intrafusal muscle demonstrated by in situ hybridization analysis (data not shown). However, at P14, when the NT3 ${ }^{F L O X / F L O X} / m y f 5^{\mathrm{CRE}}$ animals exhibit a motor phenotype similar to the NT3 $\Delta$ and ErbB2 $\Delta$ animals, the amplitude of the potentials was significantly reduced (Fig. 7I,J). At P5, the amplitude of the potentials measured was $5.1 \pm 0.3 \mathrm{mV}$ for the NT3 $3^{\text {FLOX/FLOX }} /$ myf $5^{\mathrm{CRE}}$ animals $(n=4)$ compared with $6.4 \pm 1.1 \mathrm{mV}$ for the controls. None of these values differed statistically ( $p=0.10$, ANOVA). In contrast, at P15, the amplitude of the potentials measured was $0.47 \pm 0.14 \mathrm{mV}$ for the $N T 3^{\text {FLOX/FLOX }} /$ my $5^{\mathrm{CRE}}$ animals $(n=3)$ and $1.98 \pm 0.44 \mathrm{mV}$ for the controls $(n=3)$, a reduction of $>75 \%$, comparable with the ErbB2 $\Delta$ and NT $3 \Delta$ mutants.

These data demonstrate that spindlederived NT3 has a late role in the maintenance of functional muscle spindle afferent connections on motor neurons and that the regulation of these monosynaptic inputs by muscle spindles in the first postnatal week does not depend on NT3 expression.

\section{The functional specificity of connections between afferents and motor neurons is preserved in ErbB2 $\Delta$ animals}

In normal mice, muscle spindle afferents project monosynaptically to homonymous and some synergist motor neurons but do not make direct connections with functionally antagonist motor neurons (Mears and Frank, 1997). To examine whether this specific pattern of connectivity was preserved in the ErbB2 $\Delta$, mice we compared the sensorimotor connections between the functionally antagonist LG and TA motor neurons. Figure 8, A and $B$, illustrates EPSPs evoked in wild-type and ErbB2 $\Delta$ LG motor neurons by stimulation of homonymous and antagonist muscle nerves. As shown in the figure, the latency of the intracellularly recorded EPSP evoked by antagonist nerve stimulation was at least $5 \mathrm{~ms}$ longer than that evoked by homonymous stimulation.

The latency of the homonymously evoked EPSP was $9.4 \pm 0.6$ 
ms (range, 8.4-10.4 ms; $n=3$ ) for wildtype $\mathrm{LG}$ motor neurons and $11.4 \pm 0.7 \mathrm{~ms}$ (range, 9.9-12.8 ms; $n=4$ ) for wild type TA motor neurons (Fig. $8 C, D$ ). When the antagonist nerves were stimulated (TA nerve and recordings made in LG motor neurons; LG with recordings made in TA motor neurons), the latencies were significantly longer ( $p<0.05, t$ test). For TA to LG stimulation, the mean latency of the EPSP was $15.9 \pm 2.3 \mathrm{~ms}$ (range, 12.5-20.2 $\mathrm{ms} ; n=3$ ) and for LG to TA, it was $17.2 \pm$ $0.6 \mathrm{~ms}$ (range, 16.5-20.4 ms; $n=3$ ). Thus, in LG motor neurons, the latency of the EPSP evoked by antagonist nerve stimulation was $6.5 \mathrm{~ms}$ longer than that evoked by homonymous nerve stimulation, and, for TA motor neurons, this difference was 5.8 ms.

It has been shown recently that stimulation of muscle afferents activates a disynaptic inhibitory pathway in neonatal mouse motor neurons (Wang et al., 2008). We did not, however, observe hyperpolarizing responses in antagonist motor neurons after stimulation of antagonist muscle nerves. The responses below the baseline in the bottom panels of Figure 8, E and $F$ (antagonist stimulation), are misleading because the traces have all been adjusted to a common baseline. During 10 $\mathrm{Hz}$ stimulation, the potentials summate so that individual responses are superimposed on this tonic depolarization. We do not consider the depolarizing potentials shown in Figure $8(A, B$, and $E, F)$ to be inverted IPSPs. The chloride equilibrium potential in neonatal motor neurons of this age has been reported to be close to the resting membrane potential or slightly hyperpolarizing (Delpy et al., 2008). Moreover, using whole-cell recording, the equilibrium potential will be more negative than normal because of cellular dialysis by the low chloride electrode solution. The depolarizing potentials activated by antagonist nerve stimulation most likely reflect activation of disynaptic excitatory pathway activated in parallel to the Ia inhibitory pathway.

The same measurements performed on ErbB2 $\Delta$ animals revealed latencies of $8.6 \pm 0.2 \mathrm{~ms}$ (range, 8.3-9.1 ms; $n=3$ ) for homonymous stimulation of LG motor neurons and $10.2 \pm 0.48$ (range, 9.2-10.8 ms; $n=3$ ) for homonymous stimulation of TA motor neurons. When the antagonist nerves were stimulated, the latencies were $14.2 \pm 1.3$ (range, $11.3-20.2 \mathrm{~ms} ; n=6$ ) for LG motor neurons and $15.3 \pm 0.5$ (range, $11.1-23.4 \mathrm{~ms} ; n=6$ for TA motor neurons). None of these latencies were statistically different from the wild-type animals. For the ErbB2 $\Delta$ animals, the difference in homonymous and antagonist latency was $5.6 \mathrm{~ms}$ for LG motor

A

C

G
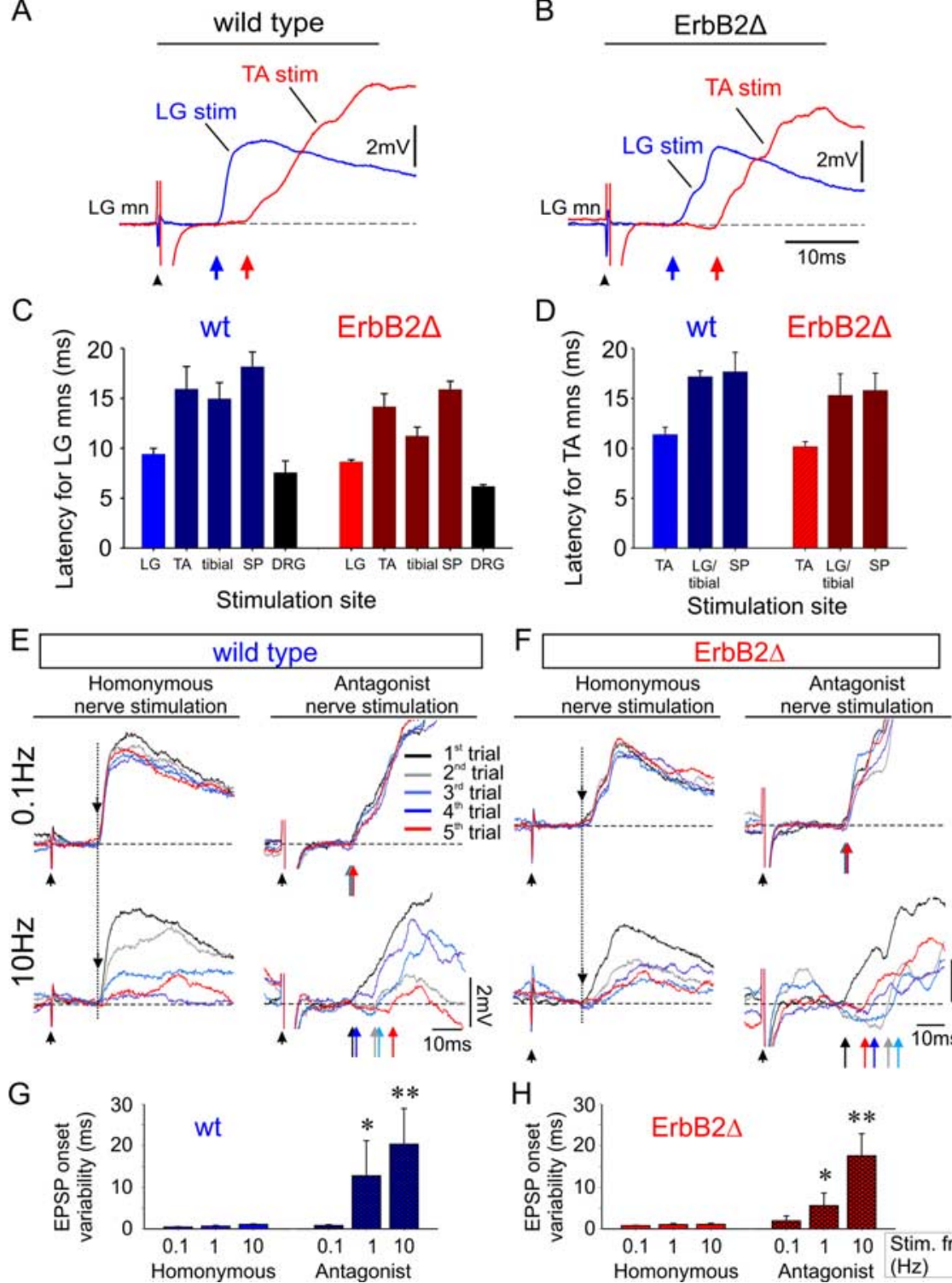

Stimulation site

Figure 8. Muscle-identified motor neurons retain specific inputs from primary afferents in ErbB2 $\Delta$ mutant mice. $A$, Comparison of the potentials evoked by homonymous (blue trace, $L G$ ) and antagonist (red trace, TA) muscle nerve stimulation at 5 T intensity averaged from five stimuli applied once every $10 \mathrm{~s}$. The blue and red arrows indicate the onset of the EPSPs. $\boldsymbol{B}$, Recordings of homonymous and antagonist evoked synaptic potentials in an ErbB2 $\Delta$ LG motor neuron. The conditions and stimulation parameters were the same as for the wild-type records in $A$. C, D, Bar graph showing the average latency of the synaptic potential in evoked in LG (C) and TA (D) motor neurons by stimulation of different nerves for wild-type (blue bars) and ErbB2 $\Delta$ (red bars) mice. The black bars show the latency of the EPSP after simulation of the DRG. Error bars are SEM. $\boldsymbol{E}$, The left panels show five superimposed intracellular responses from a wild-type $L G$ motor neuron after stimulation of the homonymous $L G$ nerve. Two different frequencies of stimulation are illustrated $(0.1 \mathrm{~Hz}$, top; and $10 \mathrm{~Hz}$, bottom). Five sequential color-coded trials are illustrated. Note that the onset of the EPSP after stimulation of the homonymous nerve results in very little variation ("jitter") in the latency at the two different frequencies (as indicated by the dotted line). In contrast, stimulation of the antagonist nerve (right panels) results in more variation in the latency particularly at $10 \mathrm{~Hz}$. The color-coded arrows denote the onset of the EPSP in each trial. $\boldsymbol{F}$, Intracellular responses from an LG ErbB2 $\Delta$ motor neuron under identical testing. Note that, at $10 \mathrm{~Hz}$, both WT and ErbB2 $\Delta$ motor neurons exhibited reduced amplitude in EPSPs as expected, presumably attributable to neurotransmitter depletion. $\boldsymbol{G}, \boldsymbol{H}$, Bar graphs for wild-type $(\boldsymbol{G})$ and $\operatorname{ErbB} 2 \Delta(\boldsymbol{H})$ motor neurons comparing the average latency jitter of the homonymous and antagonist nerve-evoked EPSPs. There was no significant difference in the latency-jitter EPSP after the homonymous nerve stimulation for either wild-type or ErbB2 $\Delta$ motor neurons at all stimulation frequencies. In contrast, stimulation of the antagonist nerve resulted in significantly more jitter at stimulation frequencies of 1 and $10 \mathrm{~Hz}\left({ }^{*} p<0.05,{ }^{* *} p<0.01\right.$, ANOVA Fisher's test). neurons and $5.1 \mathrm{~ms}$ for TA motor neurons, indicating that monosynaptic inputs from muscle afferents are functional in the ErbB2 $\Delta$ animals, and, for the TA and LG motor neurons, the specificity of the connections is preserved.

Stimulation of other nerves, including the SP or another mus- 
cular branch of the tibial nerve, also resulted in synaptic potentials in LG wild-type motor neurons whose latencies were significantly longer $(p<0.05)$ than those obtained with homonymous activation (tibial to LG, $14.9 \pm 1.6$, range of $12.3-17.9 \mathrm{~ms}, n=3$; SP to $L G, 18.2 \pm 1.5 \mathrm{~ms}$, range of $15.3-20.3 \mathrm{~ms}, n=3)$. Similarly, stimulation of the SP nerve in ErbB2 $\Delta$ mice resulted in EPSP latencies of $15.8 \pm 1.7 \mathrm{~ms}$ (range, $14.1-19.2 \mathrm{~ms} ; n=3$ ). These results are consistent with the idea that homonymous nerve stimulation results in monosynaptic activation of the motor neurons and antagonist stimulation results in disynaptic or multisynaptic activation in wild-type animals.

\section{Comparison of the latency jitter of homonymous and antagonist evoked EPSPs to different frequencies of afferent stimulation}

We also examined the behavior of the homonymous and antagonist evoked potentials to stimulation at different frequencies $(0.1,1$, and $10 \mathrm{~Hz})$ to confirm that the homonymously evoked EPSPs were produced monosynaptically, whereas those evoked by antagonist nerve stimulation were not. Previous studies have shown that high-frequency stimulation of the monosynaptic potential results in a progressive decline in the amplitude of the response (Fig. 8 E, F, bottom panels). However, multisynaptic potentials, including those generated by antagonist nerve stimulation, do not show a monotonic decrease in amplitude but tend to fail in an all-or-none manner as the intervening interneurons fail to reach threshold (Lee and O'Donovan, 1991; Lev-Tov and Pinco, 1992; Li and Burke, 2001). As a result, the latency of disynaptic and polysynaptic responses will show much more variability as the stimulus frequency is increased than monosynaptic responses. This is illustrated in Figure 8, $E$ and $F$, which compares the EPSPs generated by homonymous and antagonist nerve stimulation at different frequencies of stimulation for wild-type (Fig. $8 E$ ) and ErbB2 $\Delta$ (Fig. $8 F$ ) preparations. It can be seen from the figure that stimulation of the homonymous nerve results in a small latency jitter (the difference between the minimum and maximum latency) as stimulation frequency increases. However, the jitter is substantially greater for the antagonist nerve stimulation at the different frequencies. For five wild-type motor neurons, the jitter for the homonymous stimulus at the different frequencies $(0.1,1$, and $10 \mathrm{~Hz})$ was $0.4 \pm 0.1,0.7 \pm 0.2$, and $1.1 \pm$ $0.1 \mathrm{~ms}$, respectively. For antagonist stimulation, these values were $0.8 \pm 0.2,12.8 \pm 8.5$, and $20.4 \pm 8.6 \mathrm{~ms}$, respectively. The jitter was significantly different $(p<0.05)$ between homonymous and antagonist stimulation at 1 and $10 \mathrm{~Hz}$ (Fig. 8G). This clearly demonstrates a major difference in the behavior of the homonymous and antagonist EPSPs. Similar findings were made for homonymous and antagonist stimulation in ErbB2 $\Delta$ mice (Fig. $8 H)$. For homonymous stimulation, the latency jitter was $0.8 \pm$ $0.1,1.0 \pm 0.3$, and $1.1 \pm 0.3 \mathrm{~ms}$ in six motor neurons for stimulation at the three different frequencies. For antagonist stimulation, these values were $1.9 \pm 1.2,5.6 \pm 3$, and $15.6 \pm 5.3 \mathrm{~ms}$, respectively, in six motor neurons. The difference in latency jitter between homonymous and antagonist stimulation was significant $(p<0.05)$ at 1 and $10 \mathrm{~Hz}$. There was no difference in the jitter at the different frequencies between the wild-type and ErbB2 $\Delta$ animals (ANOVA on ranks).

The anatomical specificity of the connections between muscle afferents projecting to agonist and antagonist motor neurons is preserved in $\operatorname{ErbB} 2 \Delta$ animals

Our data demonstrate that the specificity of functional connections between muscle afferents and motor neurons is preserved in the ErbB $2 \Delta$ mutant. However, because the monosynaptic EPSP in these animals is so dramatically diminished, it is possible that aberrant projections are formed in the mutant but are too weak to detect physiologically. To address this possibility, we examined the anatomical specificity of the projections in six P14 mice (three wild type mice; three ErbB2 $\Delta$ mice) by labeling the afferents from the TA and the LG muscle using CTb conjugated to either Alexa 488 (for TA) or Alexa 647 (for LG; see Materials and Methods). To assess the specificity of the projection, we counted the number of VGluT1 ${ }^{+}$boutons that were labeled with either tracer on muscle identified motor neurons. As illustrated in Figure 9, the tracer labeling is punctate so that axons and dendrites cannot be followed in continuity (supplemental Fig. 3, available at www. jneurosci.org as supplemental material). Therefore, it was necessary to label motor neurons using Texas Red Dextran, which fills the cytoplasm of the soma and proximal 50-100 $\mu \mathrm{m}$ of the dendrites. This was essential to distinguish between the cytoplasmic $\mathrm{CTb}$ and that from afferent terminals located on the dendritic surface (Fig. 9E, F). Although we occasionally observed VGlut $1^{+}$ boutons labeled with both $\mathrm{CTb}$ tracers, the overwhelming majority of VGlut ${ }^{+}$boutons were labeled with a single tracer.

To quantify the number of muscle identified synaptic $\left(\right.$ VGlut $1^{+}$) boutons on motor neuron dendrites, we examined three TA and three LG motor neurons that were labeled with a single CTb and also with Texas Red Dextran. To quantify the number of labeled afferent terminals, we chose dendrites on which at least one VGluT1 ${ }^{+}$bouton also labeled with a single CTb (488 for TA or 647 for LG motor neurons) (Fig. 9E, F). We first counted the number of VGlut $1^{+}$boutons apposed to the motor neuron dendrite over 51-117 $\mu \mathrm{m}$ (six motor neurons in three mice). There were $5.6 \pm 0.9$ boutons per $50 \mu \mathrm{m}$ of dendritic length for three LG control motor neurons and 7.2 \pm 0.6 boutons per $50 \mu \mathrm{m}$ of dendritic length for three TA motor neurons. Because there was no statistical difference between number of TA and LG motor neurons ( $p=0.24, t$ test), we pooled the data to give $6.4 \pm 0.6$ boutons per $50 \mu \mathrm{m}$ of dendritic length for the wild-type mice. Approximately $15 \pm 2.3 \%$ of these boutons were also labeled with one of the CTb tracers (Fig. 9E). In every case, the tracer was the same as that labeling the motor neuron. We found no examples of inappropriate projections (e.g., TA boutons on an LG motor neuron or vice versa).

We made similar observations in the ErbB2 $\Delta$ mutant motor neurons. We analyzed seven LG motor neurons with dendrites spanning 39-107 $\mu \mathrm{m}$. The frequency of VGluT1 ${ }^{+}$boutons on dendrites was $5.5 \pm 0.9$ per $50 \mu \mathrm{m}$ of dendritic length, and, of these, $25.8 \pm 5.3 \%$ were labeled with the appropriate CTb for the motor neuron (Fig. $9 F$ ). As with the wild-type animals, we found no instances of inappropriate labeling. There was no statistical difference in the proportion of double-labeled VGluT1 boutons between the wild-type and ErbB2 $\Delta$ mice ( $p=0.11, t$ test).

In two of six wild-type motor neurons and in four of seven ErbB2 $\Delta$ motor neurons, we observed VGlut1 ${ }^{+}$boutons labeled with the appropriate tracer on the somata. As with the dendrites, we found no inappropriately labeled boutons on the motor neuron somata.

\section{Discussion}

This study has revealed a limited role for spindle-derived factors in the development of sensorimotor connections. In the widespread absence of their targets, muscle afferents project monosynaptically to motor neurons in approximately normal numbers and with appropriate specificity, although the connections are significantly weaker than normal. During the first postnatal week, 

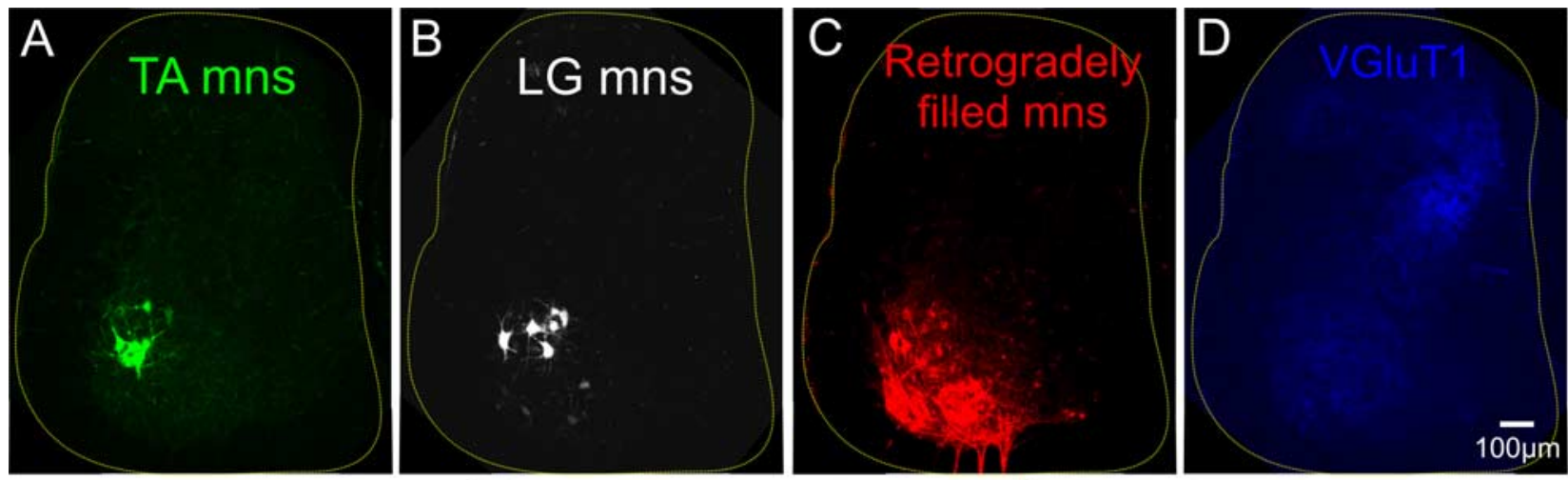

\section{Control \\ LG mn}
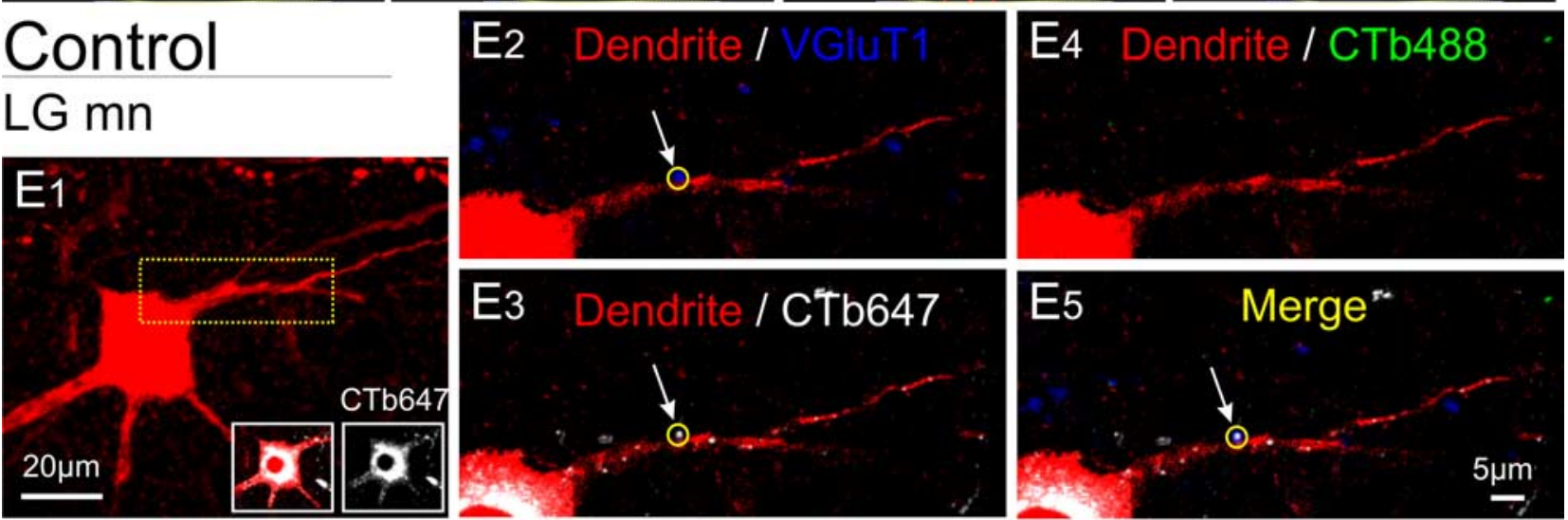

\section{$\operatorname{ErbB2} \Delta$ \\ LG $\mathrm{mn}$}
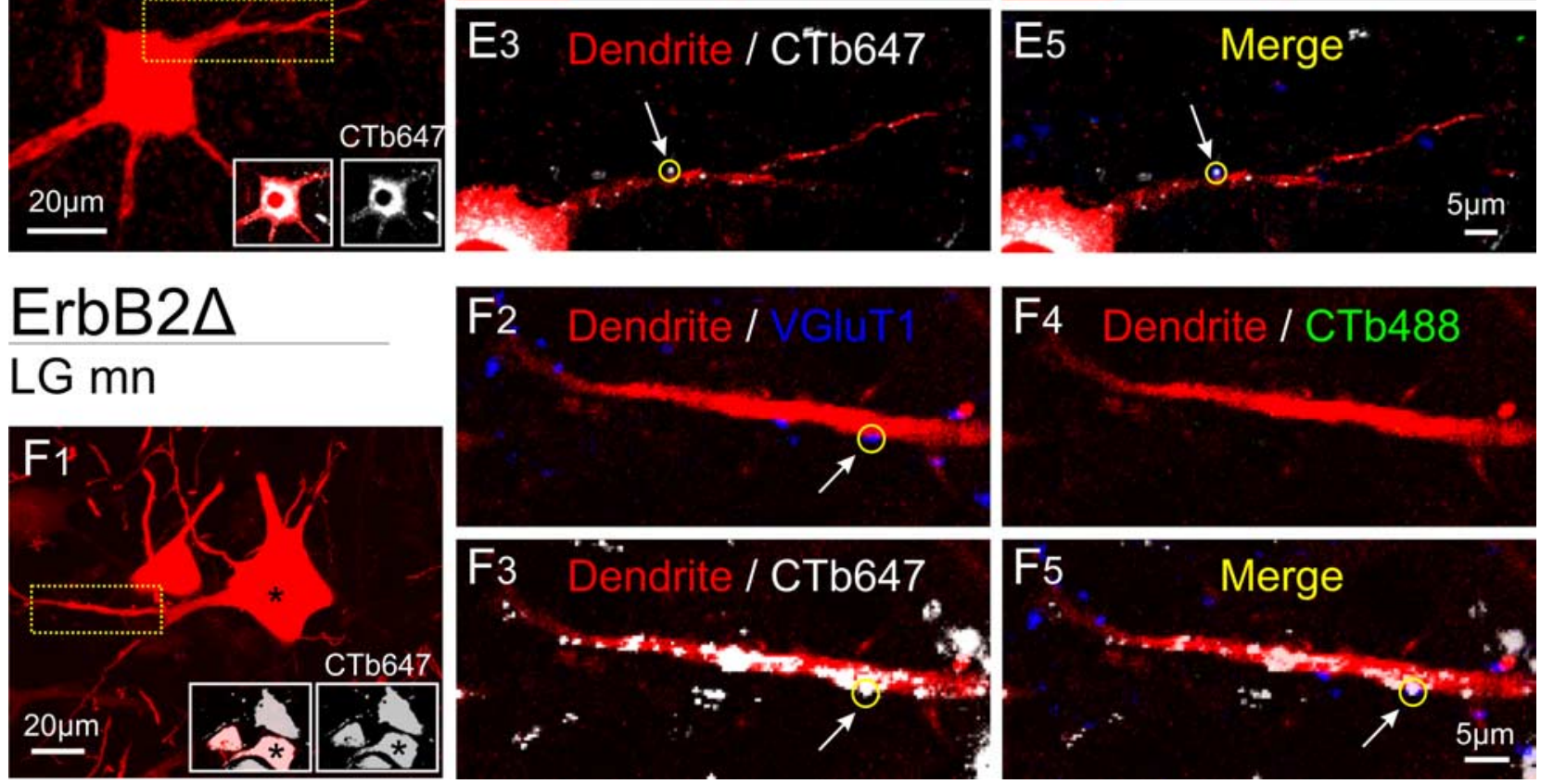

Figure 9. Motor neurons receive primary afferent boutons from homonymous but not from antagonistic muscle afferents in control and ErbB2 $\Delta$ mice. $A-D$, Confocal images from the same transverse section of a hemisected wild-type spinal cord in the $L 4 / L 5$ region showing retrograde labeling of TA motor neurons with CTb488 ( $A$, in green), $L G$ motor neurons labeled with CTb- 647 ( $\boldsymbol{B}$, in white), motor neurons (mns) retrogradely labeled with Texas Red Dextran ( $\boldsymbol{C}$, in red), and VGluT1 immunoreactivity ( $\boldsymbol{D}$, in blue). The images in the $\boldsymbol{E}$ series are from a wild-type $L \mathbf{G}$ motor neuron E1, A z-stack projection (total scan depth, $9.1 \mu \mathrm{m}$ ) of the motor neuron retrogradely labeled with Texas Red Dextran. The insets show the soma of the motor neuron double labeled with Texas Red Dextran and (Tb- 647 (red and white) and labeled with CTb- 647 alone (white), confirming the identity of the motor neuron as $\mathrm{LG}$. The dotted yellow rectangle is shown at higher magnification with various combinations of fluorochromes in the E2-E4 series. Each image is a single optical scan (0.69 $\mu \mathrm{m}$ thickness). E2 shows VGluT1 immunoreactivity (blue) and the Texas Red Dextran-labeled dendrite of the $L G$ motor neuron. The circle identifies a putative primary afferent bouton labeled with VGluT1, which is also labeled with (Tb- 647 (E3) as seen in the merged image (E5), indicating that it is a bouton from an afferent originating in the LG muscle. $E 4$, No VGut1 ${ }^{+}$boutons were labeled with the tracer (CTb488, green) from the antagonist muscle (TA). The series of images $F 1-F 5$ are from an LG ErbB2 motor neuron and were acquired in the same way as those of the images shown in E1-E5.

NT3 from nonspindle sources or spindle-derived factors other than NT3 must regulate the strength of the connections because the amplitude of muscle afferent evoked ventral root potentials was normal in animals in which NT3 was genetically eliminated from spindles. By the second postnatal week, however, the strength of the connections becomes dependent on spindle-derived NT3.

Why are the afferent connections weaker in the ErbB2 $\Delta$ mutants?

We found that the amplitude of muscle afferent-evoked synaptic potentials was reduced by nearly $80 \%$ in the ErbB $2 \Delta$ animals. If muscle afferent cell death increased in the ErbB2 $\Delta$ mutant, it could result in fewer sensorimotor connections. This explanation seems unlikely, however, because there is no obvious loss of largediameter parvalbumin ${ }^{+}\left(\mathrm{PV}^{+}\right)$cell bodies in the dorsal root ganglia at P5 in the ErbB2 $\Delta$ mutant (supplemental Fig. 5, available at www.jneurosci.org as supplemental material) (Leu et al., 2003, their Fig. 7), and the density of $\mathrm{PV}^{+}$afferent central projections appeared normal at P6 (Fig. $2 B, E$ ).

Another possibility is that fewer afferents form functional connections with motor neurons than normal. This might occur if the only afferents making functional connections with motor 
neurons were those innervating rudimentary spindles. We estimate the number of rudimentary spindles in the mutant TA muscle to be approximately one-third of the normal number of spindles in the TA muscle. This figure approximates the fraction of the control monosynaptic response $(\sim 22 \%)$ recorded in the ErbB2 $\Delta$ animals and is consistent with the idea that only afferents innervating spindles make functional connections. Interestingly, if this does explain the reduced connection strength, then most afferent synapses on motor neurons $(\sim 67 \%)$ must be functionally silent because the number of VGluT $1{ }^{+}$terminals on motor neuron somata at P21 and the initial $40-100 \mu \mathrm{m}$ of motor neuron dendrites at P14 was not reduced proportionately. However, if afferent input was selectively lost from the distal dendrites, which we could not assess, this latter conclusion would have to be modified.

The apparent mismatch between the number of VGluT1 ${ }^{+}$ terminals and the amplitude of the muscle-nerve-evoked EPSP in the ErbB2 $\Delta$ animals might also reflect some abnormality in the function of the afferent boutons. Ultrastructural studies of individual Ia afferent boutons have revealed that vesicle number, active zone area, and active zone number are highly variable among Ia muscle-spindle afferent boutons on adult cat motor neurons (Pierce and Mendell, 1993). If any of these characteristics were reduced in the ErbB2 $\Delta$ terminals, it could account for the reduction in the amplitude of the EPSPs.

The reduced strength of the connections in the $\operatorname{ErbB} 2 \Delta$ animals could occur because the spindle-derived signals regulating the strength of the central connections are not expressed in the rudimentary ErbB2 $\Delta$ spindles. Accordingly, muscle afferents would still form connections with motor neurons, whether or not the afferent innervated a rudimentary intrafusal fiber, but the amplitude of the monosynaptic EPSP from each afferent would be reduced. Our data do not address this issue, but it could be studied by examining the potentials generated in ErbB2 $\Delta$ motor neurons by single muscle afferents (Lichtman and Frank, 1984).

One issue outside the scope of the present work is the nature of the synaptic defect in the mutant animals. Although we cannot resolve this question without additional study, in preliminary findings, we observed that the sensorimotor potentials recorded in $\operatorname{ErbB} 2 \Delta$ motor neurons appeared to exhibit less synaptic depression during high-frequency stimulation than those recorded in the wild-type controls (supplemental Fig. 4, available at www.jneurosci.org as supplemental material). In normal animals, the degree of high-frequency-dependent synaptic depression of the monosynaptic EPSP is believed to be proportional to the probability of transmitter release (Lev-Tov and Pinco, 1992). This raises the possibility that the decreased amplitude of afferent-evoked monosynaptic potentials in the ErbB2 $\Delta$ mice might be attributable to a reduced probability of transmitter release.

It is instructive to compare our results with the effects of neonatal axotomy, a procedure that reproduces some of the pathology present in the ErbB2 $\Delta$ animals. In normal animals, neonatal axotomy is accompanied by afferent (Himes and Tessler, 1989) and motor neuron cell death (Lowrie et al., 1987; Mentis et al., 2007), confounding an analysis of sensorimotor connectivity. However, in Bax knock-out mice, axotomy-induced cell death is greatly reduced (Kinugasa et al., 2006; Sun and Oppenheim, 2003), allowing sensorimotor connectivity to be studied. In such mice, a week after unilateral sciatic nerve transection, the amplitude of afferent evoked ventral root potentials is significantly reduced (to $16.6 \%$ of the uncut control side amplitude) and the afferent conduction is slowed (Kinugasa et al., 2006). Thus, the changes in the amplitude of the afferent-evoked synaptic potentials are similar those in ErbB2 $\Delta$ mice, although the reduced afferent conduction velocity is not. Additional study of the sensorimotor connections in this mouse might be useful in isolating the effects of afferent disconnection from the periphery from other abnormalities present in the ErbB2 $\Delta$ mice.

Our data reveal that, when spindle NT3 is selectively eliminated, the amplitude of afferent-evoked ventral root potentials recorded at P5 is similar in wild-type and NT3 $\Delta$ mice. This indicates that the reduced amplitude of afferent-evoked monosynaptic EPSPs observed at P4-P5 in the ErbB2 $\Delta$ mice is not attributable to the absence of muscle spindle-derived NT3. Rather, spindle-derived NT3 is required later in the development to maintain the functional competence of the synapses between muscle spindle afferents and motor neurons, and its loss does not become physiologically evident until the second postnatal week.

The mechanism by which spindle-derived NT3 modulates central synapses during development is unknown. In the rat, Mendell and his colleagues have shown in vitro that exogenous NT3 can acutely potentiate the monosynaptic connections between spindle afferents and motor neurons in the first postnatal week (Arvanov et al., 2000; Arvanian and Mendell, 2001) and that chronic exposure to NT3 also potentiates the connections by a separate mechanism (Arvanian et al., 2003). The acute effects of NT3 disappear by the second postnatal week, suggesting a change in the role of NT3 in sensorimotor synaptic function after P7. The apparent absence of any changes in the strength of sensorimotor connections at P5 in the NT3 $\Delta$ mutant raises the possibility that the afferents are deriving NT3 from another source. Motor neurons are one potential source of NT3 (Schecterson and Bothwell, 1992; Buck et al., 2000), although in the absence of spindlederived NT3, alternative sources of NT3 are apparently insufficient to maintain the strength of the connections in the second postnatal week.

In wild-type mice, the amplitude of the Ia EPSP remains constant during the first two postnatal weeks (Li and Burke, 2002) despite the continued expansion of the motor neuron dendritic tree and the addition of new afferent synapses. It is conceivable that NT3 may be involved in establishing the constancy of the postsynaptic response to muscle afferent stimulation in the face of these anatomical changes.

\section{The functional specificity of monosynaptic afferent inputs on motor neurons is maintained in the absence of normal muscle spindle development}

Evidence from several vertebrate species suggests that the specificity of connections between muscle spindle afferents and motor neurons is regulated by factors originating in the limb, possibly from muscle spindles (Smith and Frank, 1988; Wenner and Frank, 1995). Our findings suggest that the peripheral signal regulating afferent-motor neuron specificity is not derived from the muscle spindle because the specificity of connections between muscle afferents and motor neurons is preserved in animals lacking the majority of intrafusal muscle fibers.

As we have argued above, our data suggest that afferents can make anatomical connections with motor neurons whether or not they retain a connection to the rudimentary muscle spindle. If the spindle connection determined the specificity of the central projections to motor neurons, then afferents disconnected from their spindle would lack specificity and make erroneous connections. However, because we did not observe erroneous connections either physiologically or anatomically, it suggests that afferents disconnected from the spindle make appropriate con- 
nections. The anatomical analysis of specificity is limited because we could only examine muscle afferent boutons on the soma and the initial $40-100 \mu \mathrm{m}$ of the dendrite. It remains possible that erroneous connections are made on more distal parts of the dendritic tree that are either silent or generate synaptic potentials too small to be detectable in our experiments. It is also possible that factors diffusing from the rudimentary spindles could determine specificity without requiring a direct connection between the afferent and spindle. However, it seems reasonable to assume that such signaling would be greatly reduced in the mutant animals, which retain $<10 \%$ of the normal number of intrafusal fibers.

The source of peripheral, instructive signals that regulate afferent specificity remain to be identified. It is known that prenatal overexpression of NT3 by muscle disrupts the specification of muscle spindle afferent connections with motor neurons (Wang et al., 2007) and that peripheral glial cell line-derived neurotrophic factor regulates sensorimotor connections in distinct cervical spinal motor pools (Vrieseling and Arber, 2006). Whatever the source of the signal, our experiments suggest that distinct aspects of the periphery control specific features of the synaptic connections between muscle afferents and motor neurons. Functional maturation of the connections requires the spindles, but establishing the specific pattern of connectivity does not.

\section{References}

Andrechek ER, Hardy WR, Girgis-Gabardo AA, Perry RL, Butler R, Graham FL, Kahn RC, Rudnicki MA, Muller WJ (2002) ErbB2 is required for muscle spindle and myoblast cell survival. Mol Cell Biol 22:4714-4722.

Arber S, Ladle DR, Lin JH, Frank E, Jessell TM (2000) ETS gene Er81 controls the formation of functional connections between group Ia sensory afferents and motor neurons. Cell 101:485-498.

Arvanian VL, Mendell LM (2001) Removal of NMDA receptor $\mathrm{Mg}^{2+}$ block extends the action of NT-3 on synaptic transmission in neonatal rat motor neurons. J Neurophysiol 86:123-129.

Arvanian VL, Horner PJ, Gage FH, Mendell LM (2003) Chronic neurotrophin-3 strengthens synaptic connections to motor neurons in the neonatal rat. J Neurosci 23:8706-8712.

Arvanov VL, Seebach BS, Mendell LM (2000) NT-3 evokes an LTP-like facilitation of AMPA/kainate receptor-mediated synaptic transmission in the neonatal rat spinal cord. J Neurophysiol 84:752-758.

Bates B, Rios M, Trumpp A, Chen C, Fan G, Bishop JM, Jaenisch R (1999) Neurotrophin-3 is required for proper cerebellar development. Nat Neurosci 2:115-117.

Britsch S (2007) The neuregulin-I/ErbB signaling system in development and disease. Adv Anat Embryol Cell Biol 190:1-65

Buck CR, Seburn KL, Cope TC (2000) Neurotrophin expression by spinal motoneurons in adult and developing rats. J Comp Neurol 416:309-318.

Burke RE, Glenn LL (1996) (1996) Horseradish peroxidase study of the spatial and electrotonic distribution of group Ia synapses on type-identified ankle extensor motor neurons of the cat. J Comp Neurol 372:465-485.

Chen HH, Tourtellotte WG, Frank E (2002) Muscle spindle-derived neurotrophin 3 regulates synaptic connectivity between muscle sensory and motor neurons. J Neurosci 22:3512-3519.

Chen HH, Hippenmeyer S, Arber S, Frank E (2003) Development of the monosynaptic stretch reflex circuit. Curr Opin Neurobiol 13:96-102.

Copray JC, Brouwer N (1994) Selective expression of neurotrophin-3 messenger RNA in muscle spindles of the rat. Neuroscience 63:1125-1135.

Crone SA, Negro A, Trumpp A, Giovannini M, Lee KF (2003) Colonic epithelial expression of ErbB2 is required for postnatal maintenance of the enteric nervous system. Neuron 37:29-40.

Delpy A, Allain AE, Meyrand P, Branchereau P (2008) NKCC1 cotransporter inactivation underlies embryonic development of chloridemediated inhibition in mouse spinal motoneuron. J Physiol 586:1059-1075.

Fariñas I, Jones KR, Backus C, Wang XY, Reichardt LF (1994) Severe sensory and sympathetic deficits in mice lacking neurotrophin-3. Nature 369:658-661.

Frank E, Wenner P (1993) Environmental specification of neuronal connectivity. Neuron 10:779-785.
Himes BT, Tessler A (1989) Death of some dorsal root ganglion neurons and plasticity of others following sciatic nerve section in adult and neonatal rats. J Comp Neurol 284:215-230.

Hippenmeyer S, Shneider NA, Birchmeier C, Burden SJ, Jessell TM, Arber S (2002) A role for neuregulin1 signaling in muscle spindle differentiation. Neuron 36:1035-1049.

Honda CN (1995) Differential distribution of calbindin-D28k and parvalbumin in somatic and visceral sensory neurons. Neuroscience 68:883-892.

Jessen KR, Mirsky R (2005) The origin and development of glial cells in peripheral nerves. Nat Rev Neurosci 6:671-682.

Kinugasa T, Kudo N, Ozaki S (2006) Peripheral targets influence sensorymotor connectivity in the neonatal spinal cord: sciatic nerve axotomy in Bax-deficient mice. Neurosci Res 54:30-37.

Ladle DR, Pecho-Vrieseling E, Arber S (2007) Assembly of motor circuits in the spinal cord: driven to function by genetic and experience-dependent mechanisms. Neuron 56:270-283.

Lee KF, Simon H, Chen H, Bates B, Hung MC, Hauser C (1995) Requirement for neuregulin receptor erbB2 in neural and cardiac development. Nature 378:394-398.

Lee MT, O’Donovan MJ (1991) Organization of hindlimb muscle afferent projections to lumbosacral motor neurons in the chick embryo. J Neurosci 11:2564-2573.

Leu M, Bellmunt E, Schwander M, Fariñas I, Brenner HR, Müller U (2003) ErbB2 regulates neuromuscular synapse formation and is essential for muscle spindle development. Development 130:2291-2301.

Lev-Tov A, Pinco M (1992) In vitro studies of prolonged synaptic depression in the neonatal rat spinal cord. J Physiol 447:149-169.

Lichtman JW, Frank E (1984) Physiological evidence for specificity of synaptic connections between individual sensory and motor neurons in the brachial spinal cord of the bullfrog. J Neurosci 4:1745-1753.

Li Y, Burke RE (2001) Short-term synaptic depression in the neonatal mouse spinal cord: effects of calcium and temperature. J Neurophysiol 85:2047-2062.

Li Y, Burke RE (2002) Developmental changes in short-term synaptic depression in the neonatal mouse spinal cord. J Neurophysiol 88:3218-3231.

Lowrie MB, Krishnan S, Vrbová G (1987) Permanent changes in muscle and motoneurones induced by nerve injury during a critical period of development of the rat. Dev Brain Res 310:91-101.

Mears SC, Frank E (1997) Formation of specific monosynaptic connections between muscle spindle afferents and motor neurons in the mouse. J Neurosci 17:3128-3135.

Mentis GZ, Alvarez FJ, Bonnot A, Richards DS, Gonzalez-Forero D, Zerda R, O'Donovan MJ (2005) Noncholinergic excitatory actions of motor neurons in the neonatal mammalian spinal cord. Proc Natl Acad Sci U S A 102:7344-7349.

Mentis GZ, Díaz E, Moran LB, Navarrete R (2007) Early alterations in the electrophysiological properties of rat spinal motoneurones following neonatal axotomy. J Physiol 582:1141-1161.

Nishimaru H, Restrepo CE, Ryge J, Yanagawa Y, Kiehn O (2005) Mammalian motor neurons corelease glutamate and acetylcholine at central synapses. Proc Natl Acad Sci U S A 102:5245-5249.

Oliveira AL, Hydling F, Olsson E, Shi T, Edwards RH, Fujiyama F, Kaneko T, Hökfelt T, Cullheim S, Meister B (2003) Cellular localization of three vesicular glutamate transporter mRNAs and proteins in rat spinal cord and dorsal root ganglia. Synapse 50:117-129.

Pierce JP, Mendell LM (1993) Quantitative ultrastructure of Ia boutons in the ventral horn: scaling and positional relationships. J Neurosci 13:4748-4763.

Scarisbrick IA, Jones EG, Isackson PJ (1993) Coexpression of mRNAs for NGF, BDNF, and NT-3 in the cardiovascular system of the pre- and postnatal rat. J Neurosci 13:875-893.

Schaeren-Wiemers N, Gerfin-Moser A (1993) A single protocol to detect transcripts of various types and expression levels in neural tissue and cultured cells: in situ hybridization using digoxigenin-labelled cRNA probes. Histochemistry 100:431-440.

Schecterson LC, Bothwell M (1992) Novel roles for neurotrophins are suggested by BDNF and NT-3 mRNA expression in developing neurons. Neuron 9:449-463.

Seebach BS, Ziskind-Conhaim L (1994) Formation of transient inappropri- 
ate sensorimotor synapses in developing rat spinal cords. J Neurosci 14:4520-4528.

Smith CL, Frank E (1988) Peripheral specification of sensory connections in the spinal cord. Brain Behav Evol 31:227-242.

Sun W, Oppenheim RW (2003) Response of motoneurons to neonatal sciatic nerve axotomy in Bax-knockout mice. Mol Cell Neurosci 24:875-886.

Tallquist MD, Weismann KE, Hellström M, Soriano P (2000) Early myotome specification regulates PDGFA expression and axial skeleton development. Development 127:5059-5070.

Tourtellotte WG, Milbrandt J (1998) Sensory ataxia and muscle spindle agenesis in mice lacking the transcription factor Egr3. Nat Genet 20:87-91.

Tourtellotte WG, Keller-Peck C, Milbrandt J, Kucera J (2001) The transcription factor Egr3 modulates sensory axon-myotube interactions during muscle spindle morphogenesis. Dev Biol 232:388-399.

Vrieseling E, Arber S (2006) Target-induced transcriptional control of dendritic patterning and connectivity in motor neurons by the ETS gene Pea3. Cell 127:1439-1452.
Wang Z, Li LY, Taylor MD, Wright DE, FrankE (2007) Prenatal exposure to elevated NT3 disrupts synaptic selectivity in the spinal cord. J Neurosci $27: 3686-3694$.

Wang Z, Li L, Goulding M, Frank E (2008) Early postnatal development of reciprocal Ia inhibition in the murine spinal cord. J Neurophysiol 100:185-196.

Wenner P, Frank E (1995) Peripheral target specification of synaptic connectivity of muscle spindle sensory neurons with spinal motor neurons. J Neurosci 15:8191-8198.

Wright DE, Zhou L, Kucera J, Snider WD (1997) Introduction of a neurotrophin-3 transgene into muscle selectively rescues proprioceptive neurons in mice lacking endogenous neurotrophin-3. Neuron 19:503-517.

Wu SX, Koshimizu Y, Feng YP, Okamoto K, Fujiyama F, Hioki H, Li YQ, Kaneko T, Mizuno N (2004) Vesicular glutamate transporter immunoreactivity in the central and peripheral endings of muscle-spindle afferents. Brain Res 1011:247-251. 